\title{
Pyramidal cell types drive functionally distinct cortical activity patterns during decision-making
}

Simon Musall ${ }^{1,2,7}$, Xiaonan R. Sun ${ }^{3,4,7}$, Hemanth Mohan ${ }^{3,5}, \mathrm{Xu} \mathrm{An}^{3,5}$, Steven Gluf ${ }^{3}$, Rhonda Drewes ${ }^{3}$, Pavel Osten ${ }^{3}$, Anne K. Churchland ${ }^{3,6}$

${ }^{1}$ Institute of Biological Information Processing (IBI-3), Forschungszentrum Jülich, Jülich, Germany

${ }^{2}$ Department of Neurophysiology, Institute of Biology 2, RWTH Aachen University, Aachen, Germany

${ }^{3}$ Cold Spring Harbor Laboratory, Neuroscience, Cold Spring Harbor, NY, USA

${ }^{4}$ Department of Neurosurgery, Zucker School of Medicine, Hofstra University, Hempstead, NY, USA

${ }^{5}$ Department of Neurobiology, Duke University Medical Center, Durham, NC

${ }^{6}$ Department of Neurobiology, David Geffen School of Medicine, University of California, Los Angeles, Los Angeles, CA, USA.

${ }^{7}$ These authors contributed equally

\begin{abstract}
Understanding how cortical circuits generate complex behavior requires investigating the cell types that comprise them. Functional differences across pyramidal neuron (PyN) types have been observed within cortical areas, but it is not known whether these local differences extend throughout the cortex, nor whether additional differences emerge when larger-scale dynamics are considered. We used genetic and retrograde labeling to target pyramidal tract (PT), intratelencephalic (IT) and corticostriatal projection neurons and measured their cortex-wide activity. Each PyN type drove unique neural dynamics, both at the local and cortex-wide scale. Cortical activity and optogenetic inactivation during an auditory decision task also revealed distinct functional roles: all PyNs in parietal cortex were recruited during perception of the auditory stimulus, but, surprisingly, PT neurons had the largest causal role. In frontal cortex, all PyNs were required for accurate choices but showed distinct choice-tuning. Our results reveal that rich, cell-type-specific cortical dynamics shape perceptual decisions.
\end{abstract}

\section{Introduction}

The neocortex is organized into discrete layers that form a vertically-arranged microcircuit motif. This core circuit is largely conserved across cortical areas with each layer consisting of distinct excitatory and inhibitory cell types that can be categorized based on genetic markers, cell morphology, anatomical projections or developmental lineage ${ }^{1}$. The precise interplay between these cell types is crucial for accurate cortical circuit function and their respective functional roles are the subject of intense study. Tremendous progress has been made particularly for cortical interneurons, where the availability of specific mouse driver lines has revealed the functional arrangement of inhibitory circuit motifs ${ }^{2-4}$, e.g. for network synchronization ${ }^{5-7}$ and state-dependent sensory processing ${ }^{8-11}$. However, the roles of glutamatergic pyramidal neuron $(\mathrm{PyN})$ types are less well established, although PyNs comprise $\sim 80 \%$ of all cortical neurons and form almost all long-range projections that enable the communication between local cortical circuits and other brain areas.

While PyNs are still often treated as a monolithic group, PyN types appear to be far more diverse than interneurons with at least one hundred putative types indicated by RNA sequencing ${ }^{12}$. These molecular signatures are critical for categorizing PyNs and go far beyond layer identity because 
different PyN types are often intermingled within layers ${ }^{13-17}$. PyNs can also be classified based on their projection target. Long-range projection neurons are broadly categorized into two major types: intratelencephalic (IT) neurons, projecting to other cortical structures and the striatum, and pyramidal tract (PT) neurons, projecting to subcortical structures, such as the superior colliculus (SC), thalamus, the pons and the striatum. Aside from long-range projection targets, PT and IT neurons also differ in their electrophysiological properties, dendritic morphology and local connectivity, and differentially respond to sensory stimulation ${ }^{15-17}$. Recent studies in sensory cortex also showed that only PT but not IT neurons are important for active perception of tactile or visual stimuli, suggesting that PT and IT neurons encode separate streams of information ${ }^{18,19}$. Similar results have been found in secondary motor cortex (M2), where specific PT neurons are involved in motor generation ${ }^{13,20}$. This suggests that the functional divergence of PyN types could be key for understanding cortical microcircuits, with PT and IT neurons forming functionally-distinct, parallel subnetworks that independently process different information. However, the functional tuning of individual PyNs in frontal cortex is still best predicted by cortical area location instead of laminar location or projection type ${ }^{21}$. Since PyN type function has only been studied in single areas, it is therefore not known whether PyN-specific subcircuits are the rule throughout the cortex or restricted to a subset of cortical regions.

An ideal method to address this question is widefield calcium imaging, which allows measuring neural activity across the dorsal cortex with cell-type specificity ${ }^{22-24}$. Indeed, interneuronspecific widefield imaging has already revealed clear differences in the spatiotemporal dynamics of different inhibitory cell types during an odor detection task ${ }^{25}$. However, cortex-wide studies of different PyN types are lacking, in part due to the limited availability of PyN-specific driver lines $^{26-28}$. Here, we used two novel knock-in mouse driver lines for PT and IT neurons ${ }^{26}$ and performed widefield imaging to measure PyN-type-specific activity while animals performed a perceptual decision-making task. Moreover, we developed a retrograde labeling approach to selectively measure the activity of corticostriatal projection (CStr) neurons throughout the dorsal cortex. Dimensionality-reduction and clustering analyses revealed that cortex-wide dynamics were clearly distinct for each PyN type, suggesting the existence of specialized subcircuits. Cortical dynamics of different PyNs were further segregated based on their role in decisionmaking, with encoder and decoder approaches revealing the strongest stimulus- and choicerelated modulation in sensory, parietal and frontal cortices. This was confirmed by PyN-typespecific inactivation experiments. In parietal cortex, PT neurons were most important for sensory processing while all PyN types in frontal cortex were needed for choice formation and retention. Taken together, our results demonstrate that different PyN-types exhibit functionally distinct, cortex-wide neural dynamics with separate roles during perceptual decision-making.

\section{Results}

To monitor PyN-type specific neural activity throughout the dorsal cortex, we used two knock-in inducible CreER lines that target developmentally-distinct classes of excitatory cortical neurons: Fezf2-CreER targeting PT neurons and PlexinD1-CreER targeting IT neurons ${ }^{26}$. For PyN-type specific GCaMP6s expression, both lines were crossed with the Ai162 reporter $\operatorname{line}^{29}$ and CreER activity was induced at four weeks of age. In each mouse line, GCaMP expression was restricted to specific cortical layers and PyN types (Fig 1a,b). In Fezf2 mice, expression was concentrated in layer 5 b (Fig. 1a). We observed axonal projections to multiple subcortical regions, such as striatum and the corticospinal tract, confirming that Fezf2 is a reliable marker for corticofugal PT neurons. In PlexinD1 mice, GCaMP expression was restricted to layer $2 / 3$ and layer $5 \mathrm{a}$ and axonal projections were visible in the corpus callosum and the striatum (Fig. 1b). These results 
are in agreement with earlier reports ${ }^{26}$, confirming that PlexinD1 is a reliable marker for intracortical and corticostriatal IT neurons.

After confirming non-overlapping expression patterns of PT and IT neurons, we measured celltype-specific cortical activity with widefield calcium imaging. All imaging data was aligned to the Allen Common Coordinate Framework v3 $(\mathrm{CCF})^{25,30}$ to compare activity across individuals and PyN types. Both lines yielded robust GCaMP-dependent fluorescence and we observed rich neural dynamics throughout the dorsal cortex (Supp. Movies 1-3). We first used retinotopic mapping to assess sensory responses of different cell types and the resulting spatial arrangement of visual areas. Both PT and IT neurons robustly responded to visual stimulation and we could construct retinotopic maps that reliably indicated the location of known visual areas (Fig. 1c) ${ }^{31}$. Retinotopic maps were similar to those observed in an Emx-Cre-Ai93 mouse line (EMX) that expressed GCamp6 $\mathrm{f}$ in all excitatory cortical neurons ${ }^{32}$, suggesting that the functional architecture of visual areas is comparable across PyN types. However, clear differences were apparent in the modulation of cortical regions in the absence of visual stimulation. For example, the total variance of cortex-wide activity was largest in parietal and frontal regions in PT neurons (Fig. 1d, left) while variance was highest in visual and somatosensory regions of IT neurons (Fig. 1d, center). This was comparable to variance in EMX mice, which showed additional modulation in retrosplenial (RS) cortex (Fig. 1d, right). These different patterns were also highly consistent across individual mice (Supp. Fig. 1).
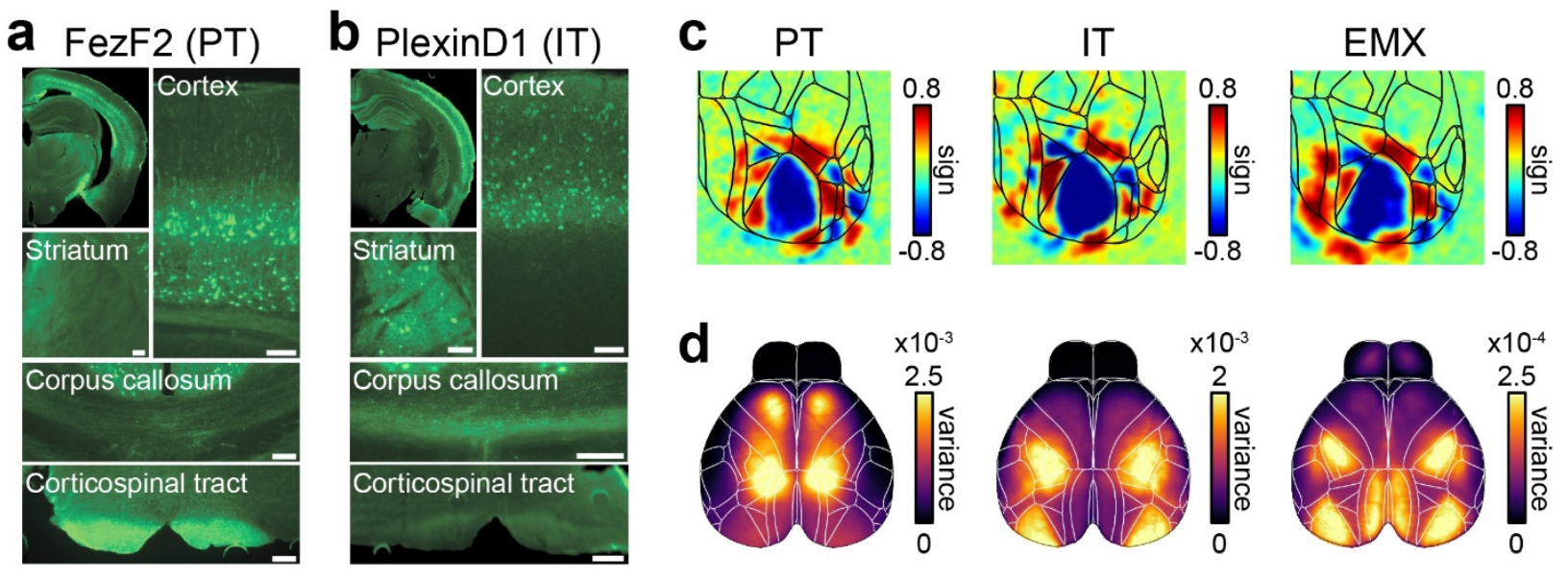

Figure 1. Knock-in mouse lines allow a PyN-type-specific view of cortex-wide neural activity.

a) GCaMP6s-expression in Fezf2-CreER-Ai162 mice was widespread throughout the dorsal cortex, and largely confined to deeper layer $5 \mathrm{~b}$. Axonal projections were found in multiple subcortical targets, such as the striatum, and the corticospinal tract. Scale bars are $100 \mu \mathrm{m}$. b) GCaMP6s-expression in PlexinD1-CreER-Ai162 mice was widespread throughout the cortex and restricted to superficial layers $2 / 3$ and layer $5 \mathrm{a}$. Subcortical axonal projections were found in striatum and corpus callosum but not the corticospinal tract. c) Visual sign maps from retinotopic mapping experiments. Both PyN types showed clear retinotopic responses in primary and secondary visual areas. Area outlines largely resembled known areas that were also observed in nonspecific EMX mice. d) Total variance maps, showing most modulated cortical regions in each PyN type.

The observed differences in variance could indicate general differences in the spatiotemporal dynamics of cortex-wide activity across PyN types. To describe such differences, we performed semi-nonnegative matrix factorization (sNMF), reducing the imaging data to a small number of spatial- and temporal components that capture the majority of all variance $(>99 \%)^{23,33}$. Here, the dimensionality (the number of required components) provides a measure of the overall complexity of cortical dynamics in each PyN type. PT neurons had the lowest dimensionality (Fig. 2a) and were also the most correlated across cortical regions (Supp. Fig. 2). 

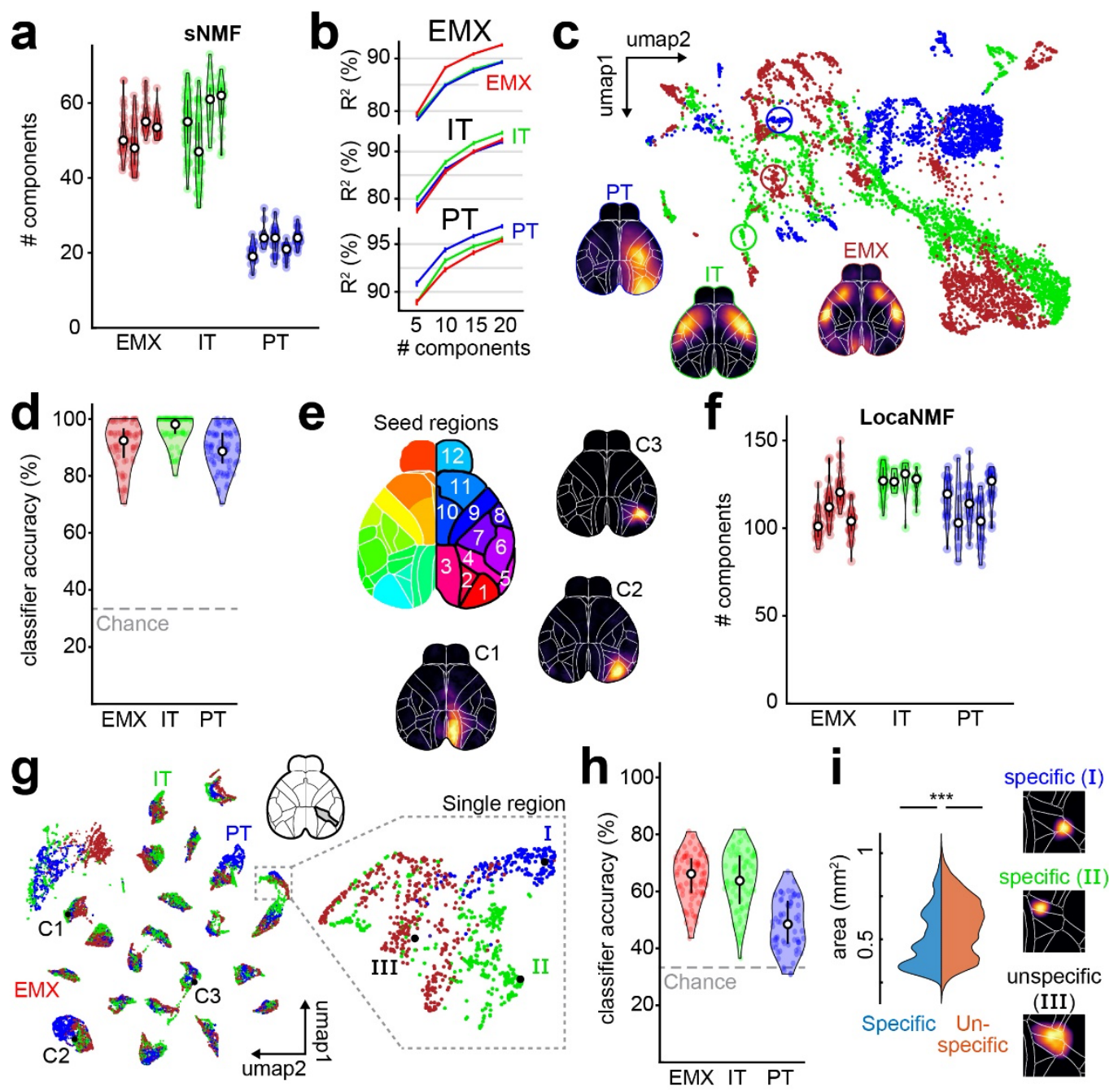

Figure 2. PyN types exhibit unique cortical activity patterns.

a) Number of sNMF components, accounting for $99 \%$ of cortical variance in each PyN type. Violin plots show individual mice in each group, dots represent individual sessions. b) $\mathrm{R}^{2}$ of EMX, IT and PT reconstructions (top to bottom panels), using components from different PyN types (red, green, and blue traces). For within-group reconstructions, only components from other mice were used. c) UMAP embedding of spatial sNMF components for EMX (red), IT (green) and PT (blue) mice. Maps show example spatial components of each type, colored circles show their respective UMAP locations. d) Accuracy of a PyN type classifier, based on neighbor identity of individual components in UMAP space. Each data point represents one session. e) Top left: Map of seed regions, used for LocaNMF analysis. Example spatial components (C1-C3) are compact and mostly confined to each seed region. f) Number of LocaNMF components, accounting for $99 \%$ of cortical variance in each PyN type.

Conventions as in (a). g) UMAP projection embedding of spatial LocaNMF components. Conventions as in (c). Left: UMAP shows clustering of LocaNMF components from similar regions. Right: Components within individual regions are further divided for different PyN types. Black dots show UMAP location of example components in (e). h) Accuracy of a PyN type classifier, based on individual LocaNMF components. Conventions as in (d). i) Left: Peak normalized distributions of area size for PyN-type-specific (blue) versus unspecific (red) LocaNMF components. Specific components are smaller than unspecific components (specific: median $=0.49 \mathrm{~mm}^{2}, \mathrm{n}=4370$ components, unspecific: median $=0.6 \mathrm{~mm}^{2}, \mathrm{n}=10978$ components; ranksum test: $\mathrm{p}<10^{-10}$ ). Right: Examples of specific (I, II) and unspecific (III) components in right parietal cortex.

Since PT neurons are a subset of EMX neurons, their lower dimensionality could indicate that PT components are also a subset of EMX components. We therefore quantified to which extent components from different PyN types are linearly overlapping or independent from each other. For each PyN type, we computed the amount of variance $\left(\mathrm{R}^{2}\right)$ that could be explained with components from other mice of either the same or different PyN type (Fig. 2b). In all groups, 5 
components were sufficient to account for a large amount of variance $(>80 \%)$, demonstrating that different PyN types have some overlap. However, similar PyN type components explained significantly more variance $\left(\Delta \mathrm{R}^{2}\right)$ as the maximal amount that could be explained by other types (EMX, red traces, mean $\Delta \mathrm{R}^{2}=3.3 \%$, $t$-test: $p<10^{-10}$; IT, green traces, mean $\Delta \mathrm{R}^{2}=1.5 \%, p<10^{-}$ ${ }^{10}$; PT, blue traces, mean $\Delta \mathrm{R}^{2}=1.2 \%, p<10^{-10}$ ). Each group therefore contains additional PyNtype-specific variance that is independent from other types. This was also visible in reconstructed data: PT components captured larger fluctuations in IT activity (Supp. Movie 4) but failed to represent spatial details in the cortical activity patterns (Supp. Fig. 3). Despite the similar dimensionality of IT and EMX activity (Fig. 2a), EMX $\Delta \mathrm{R}^{2}$ was also similar for IT and PT components $\left(\Delta \mathrm{R}^{2}\right.$ EMX-IT $=3.4 \%, \Delta \mathrm{R}^{2}$ EMX-PT $\left.=3.3 \%, p=0.1\right)$, demonstrating that EMX data was not largely dominated by either IT or PT neurons.

To directly compare spatial activity patterns, we then focused on spatial sNMF components. Spatial components are strictly positive and can be seen as a map of positively correlated cortical areas. We wondered whether these cortex-wide correlation patterns would differ between PyN types. We therefore performed a UMAP projection of the first 20 components from all recordings and PyN types, non-linearly embedding the pixels of each component in a 2dimensional space (Fig. 2c) ${ }^{34}$. If neural activity in PT, IT and EMX mice tended to exhibit similar correlation patterns, these spatial components would be mixed together. Instead, we found that components formed clusters that were largely dominated by either PT or IT neurons (green/blue dots). EMX neurons formed a third set of non-overlapping clusters, likely reflecting the combined cortical dynamics from diverse PyN types beyond PT and IT neurons that were contained in this larger group (red dots). A simple classifier could reliably identify each group with high accuracy based on the nearest UMAP neighbors from other animals, even when the classification was based on just a single spatial component (Fig. 2d). The presence of such clear clusters is striking given that the spatial components used in the analysis were pooled over many sessions and mice. This confirms that UMAP clusters reflect consistent PyN-type-specific activity patterns, rather than idiosyncratic differences within single sessions or individual mice. Taken together, these results clearly demonstrate that PyN types differ in the complexity of cortical dynamics, contain independent variance, and exhibit unique cortex-wide correlation patterns.

A potential reason why cortex-wide correlation patterns are unique to each PyN type is that each type could show particularly distinct activity in specific cortical areas, perhaps due to local variations in indicator expression patterns. This could lead to correlation patterns that are either dominated by highly active areas or where inactive areas are 'missing'. In this case, the relatively low dimensionality of PT neurons might also be due to a lower number of active cortical areas. We therefore used a localized form of sNMF (LocaNMF) ${ }^{33}$ which obtains spatial components that are dense and spatially restricted to a specific cortical "seed" region. This results in more interpretable spatial components which resemble functionally-defined cortical areas (Fig. 2e). We then isolated these localized activity patterns for each cell-type and again compared the number of components required to explain at least $99 \%$ of the variance. As expected, the required number of components was higher than with sNMF and, interestingly, was also more comparable across cell-types (Fig. 2f). This shows that cell-type specific differences in cortexwide correlation patterns are not due to a lack of activity in some cortical areas (which would have resulted in a lower number of required components, e.g. in PT mice) but PyN-type specific differences in the coordinated activation of multi-area cortical networks. Repeating the UMAP embedding on these local components also uncovered PyN-type-specific clustering (Fig. 2g), which could be used to accurately identify each PyN type (Fig. 2h). This could either be due to the presence of specific 'subregions', where PyN types are most active in smaller parts of a given 
cortical area, or larger 'superregions', where the activity of a specific PyN type extends across known area borders. We therefore compared the size of PyN-type-specific versus nonspecific components. Interestingly, specific clusters were significantly smaller than unspecific clusters (Fig. 2i), suggesting that different PyNs might be most active in distinct subregions instead of larger multi-area components. This indicates that smaller, PyN-specific subregions may reside within the coarser, traditionally-defined cortical areas.

We next sought to determine how cortical dynamics of different PyN types are related to decision-making. We therefore examined task-related activity for each PyN type in an auditory decision-making task (Fig. 3a) ${ }^{35}$. Mice initiated trials by touching small handles, which triggered the simultaneous presentation of sequences of clicking sounds to their left and right side. After a delay period, mice reported their decision by licking one of two water spouts and were rewarded for choosing the side where more clicks were presented. To reduce temporal correlations between task events, such as trial initiation and stimulus onset, the duration of the initiation, stimulus and delay periods were randomly varied across trials. In all mice, decisions varied systematically with stimulus strength (Fig. 3b) and were equally affected by click sounds throughout the stimulus period (Supp. Fig. 4).

In all PyNs, we observed broad recruitment of cortical structures, especially in parietal and frontal regions (Fig. 3c). A clear difference between PyN types occurred during the stimulus period. Here, cortical activity was uniformly suppressed in EMX mice, partially suppressed in IT mice, and uniformly increased in PT mice (Fig. 3d). In all cell types, activity was largely symmetric across the left and right hemispheres, even when only analyzing trials where stimuli were presented on the left side and the animal made a corresponding leftward choice (Fig. 3c,d). Moreover, responses to sensory stimuli were much weaker than to movement-related events, such as trial initiation or the response (Fig. 3d, gray bar versus dashed lines). A potential explanation is that lateralized, task-related activity is obscured by cortical activity due to animal movements ${ }^{32,36-38}$. 

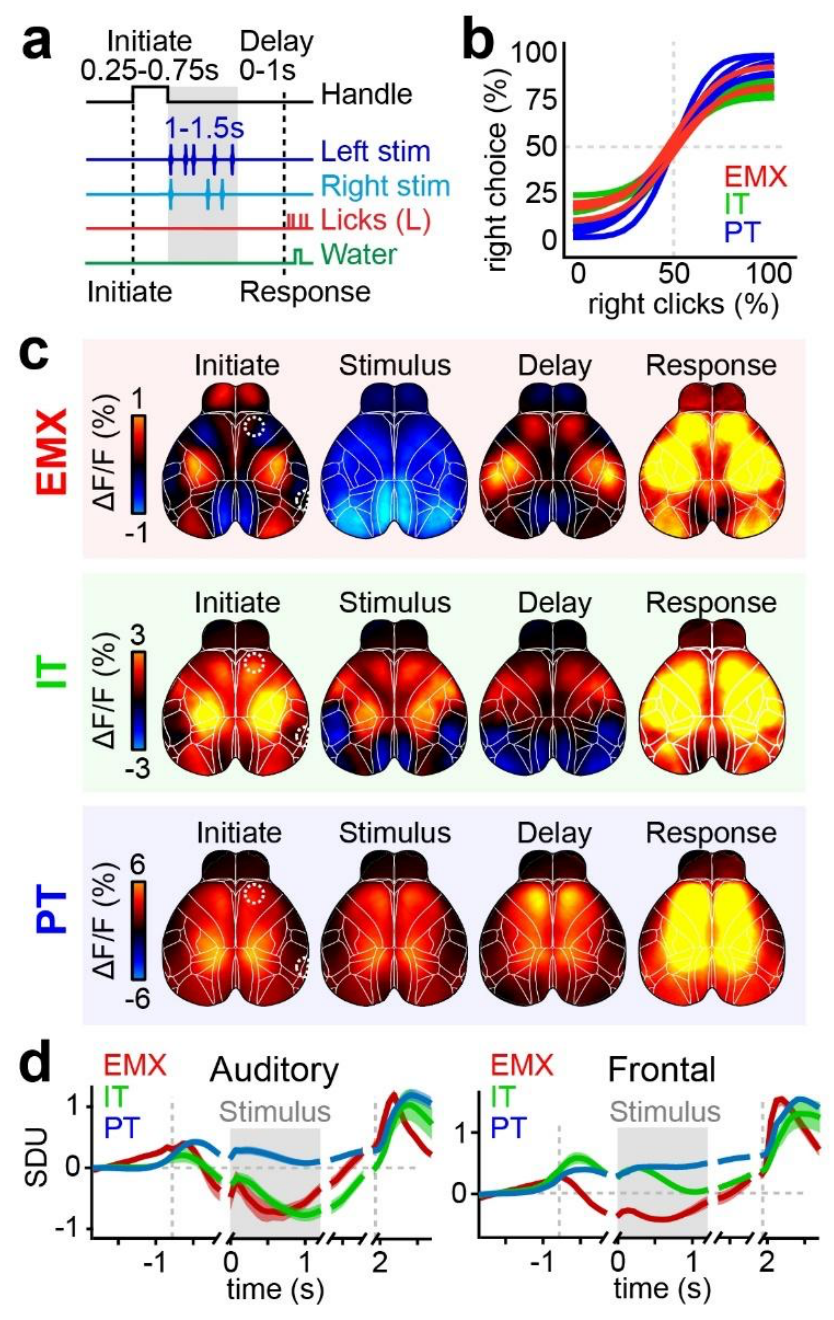

Figure 3. An auditory decision-making task reveals distinct functional activity patterns in each PyN type. a) Single-trial schematic of the auditory discrimination task. Mice touched handles to initiate randomized click sequences on the left and right side. After a delay period, a lick response on the correct side was rewarded with water. The episode duration was randomly varied in individual trials. b) Psychometric functions fit to behavioral data from the discrimination task in (a) of individual EMX (red), IT (green) and PT (blue) mice. c) Trial-averaged response maps for all correct, leftward trials in different PyN types. Shown are averages for the 'Initiation', 'Stimulus', 'Delay' and 'Response' periods shown in (a). d) Averaged activity in auditory (left) and frontal cortex (right) for each PyN type. Averages were separately aligned to each of the four trial periods, indicated by short gaps. Left dashed line: time of initiation, gray box: stimulus presentation, right dashed line: animal's response. Traces show standard deviation units (SDU). White dashed circles in (c) show respective area locations. Colors as in (b). Shading shows s.e.m.; $\mathrm{n}=4$ EMX/IT mice and 5 PT mice.

To isolate task-related activity from movements we used a linear encoding model that we recently developed ${ }^{32}$. The model predicts single-trial fluctuations in cortical activity by combining task variables, such as stimulus and choice, with movement variables, such as handle touch and licking. All variables are combined into a single design matrix; we used ridge regression to fit the model to the imaging data. We then computed the tenfold cross-validated $\mathrm{R}^{2}$ $\left(\mathrm{cvR}^{2}\right)$, to assess how well the model captured cortical activity. For all PyN types, the model successfully captured a large fraction of single-trial variance throughout the cortex (Fig. 4a). Predicted variance was higher in IT and PT mice compared to EMX mice, with over 90\% explained variance in frontal cortex of PT mice. Consistent with earlier results, a large fraction of the model's predictive power was explained by movements, which consistently captured more variance than task variables (Fig. 4b). To isolate the unique contributions of movement or task 
variables, we also computed the loss in predicted variance $\left(\Delta R^{2}\right)$ when removing either set of variables from the full model. This allowed us to separately examine their respective impact on cortex-wide activity by determining, for each PyN type, where in the cortex predictive power was lost (Fig. 4c). For movements, we observed a comparable pattern across PyN types with the highest unique contributions in primary somatosensory and motor areas (Fig. 4c, top row). In contrast, the overall pattern for task variables differed across PyN types: $\Delta \mathrm{R}^{2}$ was highest in frontal cortex of EMX and PT mice, but more diffuse in IT mice with the highest $\Delta \mathrm{R}^{2}$ in auditory cortex (Fig. 4c, bottom row). Examination of specific task variables further suggested distinct roles for each PyN type (Fig. 4d). Here, the 'choice' variable had the highest contributions in PT neurons but was overall weaker in IT neurons. Conversely, contributions from other task variables were higher in IT neurons. This dichotomy was not observed in EMX neurons, indicating that IT and PT neurons might have different functional roles that cannot be resolved without PyN-type specific measurements.
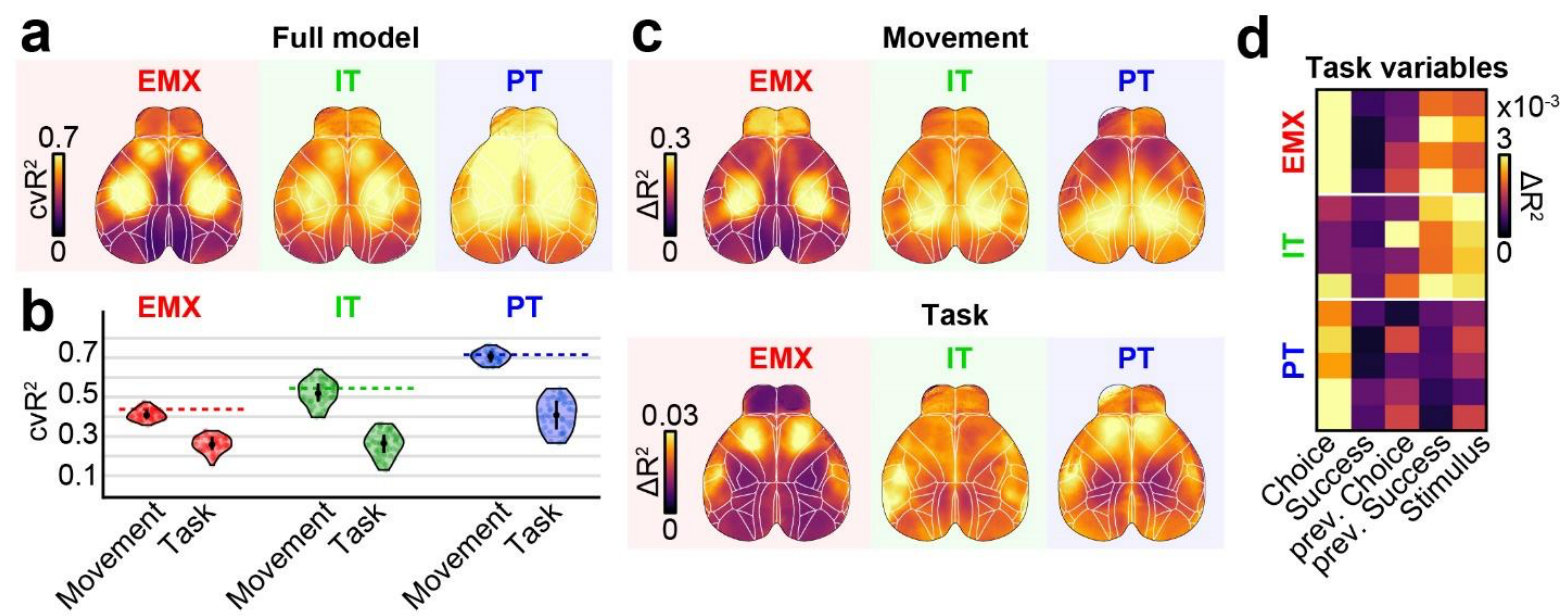

Figure 4. An encoding model uncovers task-specific differences across PyN types.

a) Average maps of $\mathrm{cvR}^{2}$ for different PyN types. The model accurately predicted cortical variance for all PyNs. b) $\mathrm{cvR}^{2}$ from two models, using only movement ('Movement') or task variables ('Task'). In all groups, movements were more predictive than task variables and accounted for the majority of the full models explained variance (dashed lines). Circles denote sessions. c) Average maps of $\Delta \mathrm{R}^{2}$ for different PyN types. Top row shows $\Delta \mathrm{R}^{2}$ for movements, the bottom $\Delta \mathrm{R}^{2}$ for task variables. Colors are scaled differently in each row. d) $\Delta \mathrm{R}^{2}$ for different task variables and PyN types, averaged over all pixels. Rows show individual mice.

To gain insight into the cortical dynamics related to stimulus and choice, we examined the event kernels learned by the model for each PyN type (see methods). Each event kernel represents the isolated time-resolved cortical response to a different task variable that is corrected for potentially confounding factors, such as movements, since those are captured by other model variables. First, we focused on responses to the auditory stimulus. In contrast to raw trial averages (Fig. 3c), the stimulus kernels in EMX mice revealed clear sensory-locked responses in auditory, parietal and frontal cortex (Fig. 5a, top). Other areas, such as somatosensory and visual cortex, were inhibited. Sensory-locked responses were also present in sensory, parietal and frontal cortex of PT and IT mice (Fig. 5a, center and bottom). However, the cortex-wide response patterns were not identical, for instance, no inhibition was apparent in PT mice. Sensory responses were particularly distinct in parietal cortex. Responses in EMX and IT mice were more anterolateral in parietal area A while PT responses were surprisingly prominent and more posteromedial at the border between area AM and RS (Fig. 5a,b). 


\section{a}
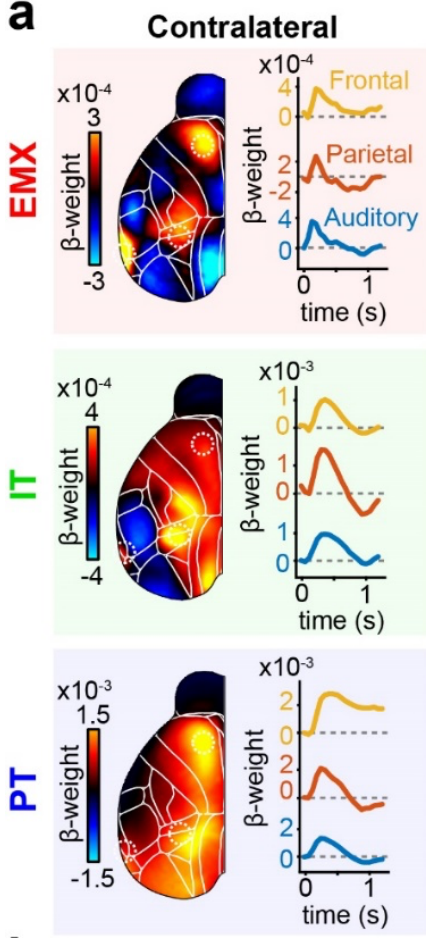

b

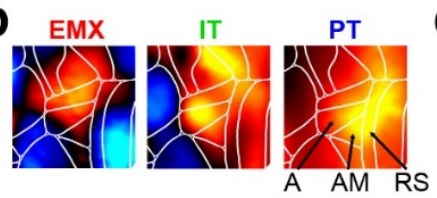

C
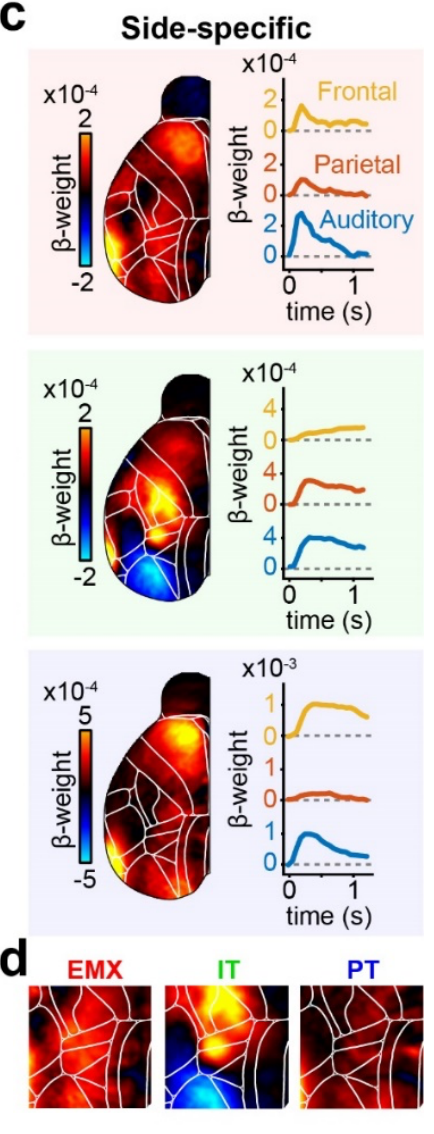

Figure 5. PyN-specific differences are evident in the location and specificity of cortical stimulus responses. a) Left: Response kernels for contralateral stimuli over all EMX (red), IT (green), and PT mice (blue), averaged between 0 and $200 \mathrm{~ms}$. Right: Stimulus-evoked activity in auditory (blue), parietal (red), and frontal cortex (yellow). Dashed circles in the left stimulus maps show locations of respective cortical areas. b) Magnified view on parietal regions of the stimulus maps in (a). PyNs differed in the location of sensory responses. Arrows show location of parietal areas A, AM and the RS. c) Side-specific stimulus responses, computed as the difference between contraand ipsilateral stimulus kernels. Conventions as in (a). d) Magnified view on parietal regions of side-specific maps in (c). IT neurons show clear, side-specific responses that were weaker in EMX and absent for PT neurons.

PyN types also differed in their response dynamics following the sensory stimulus. While some cortical areas, such as auditory cortex, preferentially responded to contralateral stimuli, PT neurons in parietal cortex showed clear bilateral responses, regardless whether the stimulus was presented to the contra- or ipsilateral side. To assess such side-specificity, we computed the difference between the contra- and ipsilateral stimulus kernels (Fig. 5c, colors indicate regions for which responses differed for contra- versus ipsilateral stimulus kernels). In EMX mice, we found side-specific responses in auditory, frontal, and to a lesser extent, parietal cortex (Fig. $5 \mathrm{c}, \mathrm{d})$. IT mice showed side-specific responses in auditory and parietal but not frontal cortex. In contrast, PT mice showed clear side-specific responses in auditory and frontal but not in parietal cortex. Such differences in unilateral versus bilateral responses in PT and IT neurons may also reflect different functional roles, with unilateral responses encoding the spatial location of sensory information and bilateral responses signaling its importance for guiding subsequent decisions.

Having identified PyN-type dependent activity for sensory stimuli, we then examined choicedependent activity and again observed differences across PyN types. In EMX mice, a number of regions showed choice-related activity, particularly in the frontal cortex, while sensory and parietal regions were only weakly modulated (Fig. 6a). We also found choice signals in 
somatosensory areas of the whiskers and nose that slowly increased over the course of the trial, even before the stimulus onset (Supp. Fig. 5a). In contrast, choice-specific activity in frontal cortex strongly increased after stimulus onset and remained elevated as the decision progressed from sampling the stimulus to the subsequent delay period (Fig. 6a, yellow trace). We found equally prominent choice signals in frontal cortex of PT mice while very little modulation was seen in IT mice (Fig. 6b, Supp. Fig. 5b,c). In EMX and PT mice, positive signals for contralateral choices were concentrated in the medial part of secondary motor cortex (M2) while parts of the primary motor cortex (M1) were inhibited. This could indicate accumulation of sensory evidence and motor preparation in M2 while inhibiting parts of M1 to prevent early lick responses ${ }^{39}$.

Although the choice kernels revealed differences between PyN types, choice-related activity only accounted for a small amount of the total neural variance (Fig. 4). Since the encoding model is designed to capture as much variance as possible, we wondered if this approach might miss subtle choice signals that are specific but low in magnitude. The encoding model's ridge penalty also enforces choice-related variance to be distributed over all correlated model variables, which could 'diffuse' weaker choice-related activity from the choice to other model kernels.

To selectively isolate all choice-related activity, we therefore built a decoding model, using a logistic regression choice classifier with L1 penalty (see Methods). In contrast to the encoding model, this decoder approach isolates the cortical signals that are best suited to predict the animal's choices, regardless of their magnitude. For all PyN types, the decoder predicted the animal's choices with high accuracy, confirming that cortical activity reliably reflects trial-bytrial choices (Supp. Fig. 6a). When analyzing the decoder weights, we found comparable patterns to the encoding model's choice kernels but with much clearer separation of cortical areas (compare top row 'Delay' in Fig. 6c to Fig. 6a, left). For all PyN types, the decoder weights identified choice signals in multiple areas of the anterior cortex that evolved during decisions (Fig. 6c). In EMX and PT mice (top and bottom rows), large parts of M2 were again highly choice predictive, including the anterior lateral motor cortex (ALM) and the medial motor cortex $(\mathrm{MM})^{21}$. M2 choice weights strongly increased immediately after stimulus onset and remained elevated during the subsequent delay period (Fig. 6c,d). Surprisingly, we also found a mild suppression of M2 in IT mice. This choice-related decrease evolved more slowly during the stimulus and delay period (Fig. 6c,d) and was more spatially restricted to ALM (Fig. 6e). In somatosensory cortex, choice signals were again visible throughout the trial (Supp. Fig. 6b) while no clear modulation was seen in parietal cortex (Fig. 6d, left). This is in line with our previous results and suggests that parietal areas are recruited during sensory processing instead of action selection or motor execution ${ }^{40,41}$. 

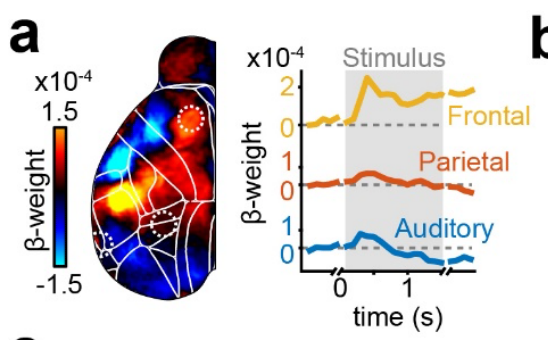

b
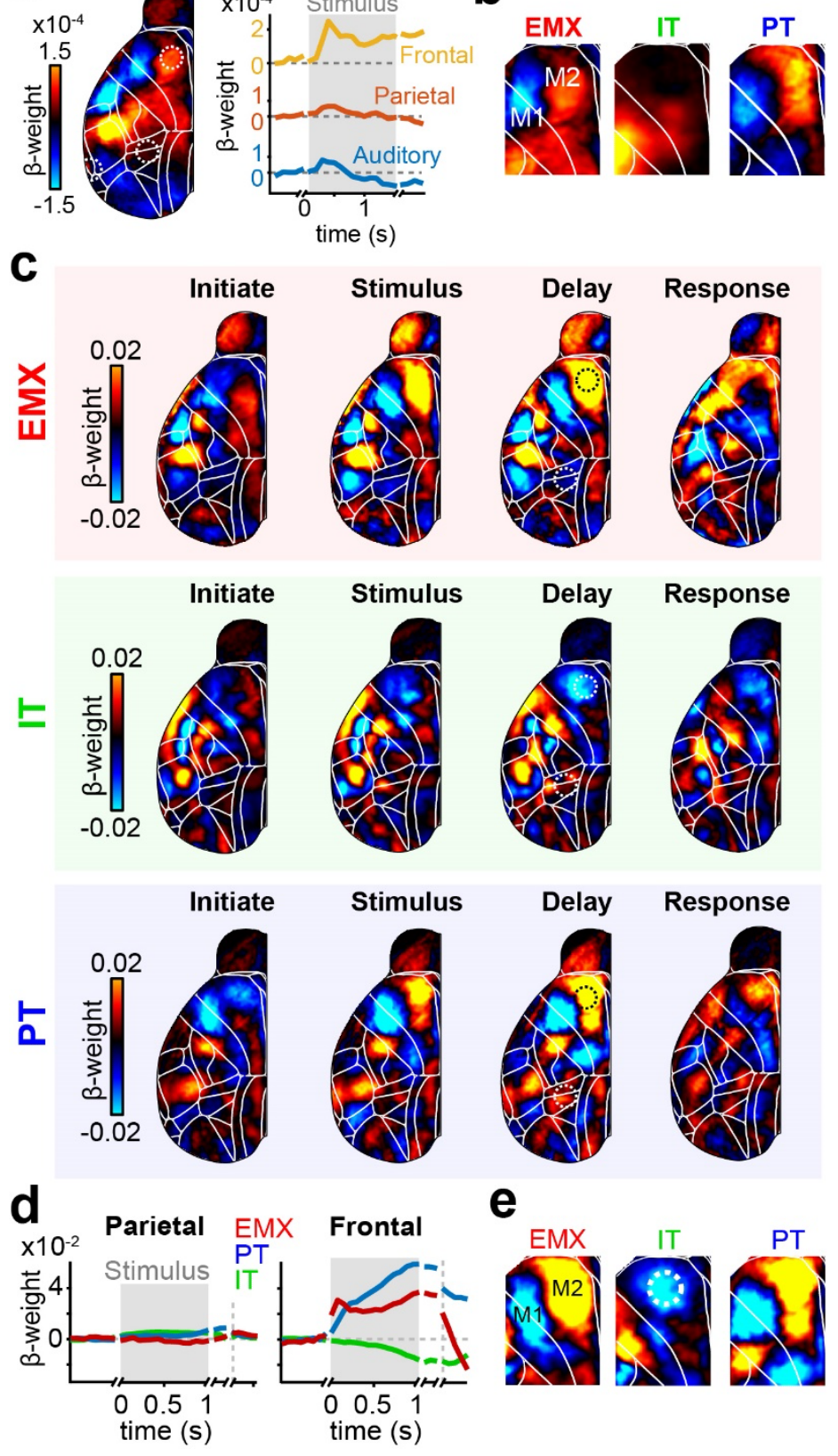

e

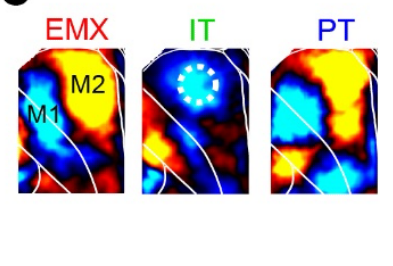

Figure 6. The temporal dynamics of choice-related activity differ across PyN types.

a) Left: Averaged choice kernels for EMX mice during the delay period. Positive weights indicate increased activity for contralateral choices, negative weights indicate choice-related reduction in activity. Right: Choice-related activity in auditory (blue), parietal (red), and frontal cortex (yellow). Traces are re-aligned to the initiation, stimulus, delay and response periods, indicated by gaps in weight traces. b) Zoomed-in map for frontal choice kernels of EMX, IT and PT mice during the delay period. c) Cortical maps of contralateral choice weights for different trial episodes. Several areas in anterior cortex showed clear choice signals. d) Baseline-corrected decoder weights in parietal (left) and frontal cortex (right) throughout the trial. Conventions as in (a). Dashed circles in the delay maps of (c) show the parietal and frontal locations that were used to compute the traces. e) Zoomed-in map for frontal decoder weights of EMX, IT and PT mice during the delay period. Dashed circle shows location of ALM.

The decoding model recovered more finely structured choice maps than the encoding model, especially in frontal cortex (compare Fig. 6e to 6b), revealing strong choice-selective activation of PT neurons but a reduction of choice weights in IT neurons. This reduction was unexpected and we wondered if it was due to the modulation of superficial intracortical projections or, instead, the deeper corticostriatal projecting IT neurons. Earlier work suggested a lack of choice 
selectivity in intracortical projection neurons ${ }^{20,21}$, and we thus hypothesized that our results in IT mice were due to corticostriatial (CStr) projecting IT neurons. To address this directly, we developed an intersectional approach that exclusively labels the majority of CStr neurons. This was achieved by performing multiple, bilateral injections of a retrograde CAV2-Cre virus in the striatum of Ai162 reporter mice (Fig. 7a), inducing wide-spread expression of GCaMP6s in CStr neurons throughout the cortex (Fig. 7b). As expected, GCaMP6 expression in CStr neurons was largely confined to layer 5 with some sparse expression in deeper layers.

We then used widefield calcium imaging to selectively measure activity from CStr neurons. As with the PT and IT mice, we obtained robust fluorescence signals throughout the dorsal cortex (Supp. Movie 5) and could identify visual areas using retinotopic mapping (Supp. Fig. 7a). sNMF analysis showed that the dimensionality of CStr mice was intermediate between PT and IT mice, and that the spatial components did not strongly overlap with other PyN types (Supp. Fig. 7b,c). This clear difference between IT and CStr mice strongly suggests that imaging signals from IT mice are not solely dominated by deeper IT-positive CStr neurons but represent a combination of superficial intracortical and deeper CStr neurons.

We then trained CStr mice in the auditory discrimination task. Trial-averaged dynamics partially resembled IT mice, for example in frontal cortex, but also showed clear differences, such as a lack of pre-stimulus suppression in sensory cortex (Fig. 7c,d; orange versus gray traces). Differences between CStr and IT mice were also visible in the stimulus kernels from the encoding model. In CStr mice, stimulus-related activity in parietal cortex was stronger than in sensory and frontal cortex but the activated parietal regions were more medial than in IT mice (Fig. 7e). Interestingly, these stimulus-driven parietal regions (Fig. 7f, top) closely resembled cortical areas that form anatomical and functional connections to the dorsomedial striatum ${ }^{42,43}$. As with PT neurons (Fig. 5b,d), parietal CStr responses equally responded to contra- and ipsilateral stimulation (Fig. 7f, bottom).

To determine whether reduced choice signals in frontal cortex of IT mice were due to CStr neurons, we then repeated the choice decoder analysis. The decoder predicted animals' choices with similarly high accuracy as for PT and IT mice (Supp. Fig. 7d). We then extracted choice weights for each task episode. Here, CStr activity was overall similar to that of IT mice, with an even stronger reduction in frontal cortex that started after stimulus onset and lasted throughout the delay and response period (Fig. $7 \mathrm{~g}$, right). As in IT mice, this effect was prominent in area ALM but did not extend to MM. Choice-specific reduction thus occurs specifically in CStr neurons in ALM and likely explains similar results in IT mice. 

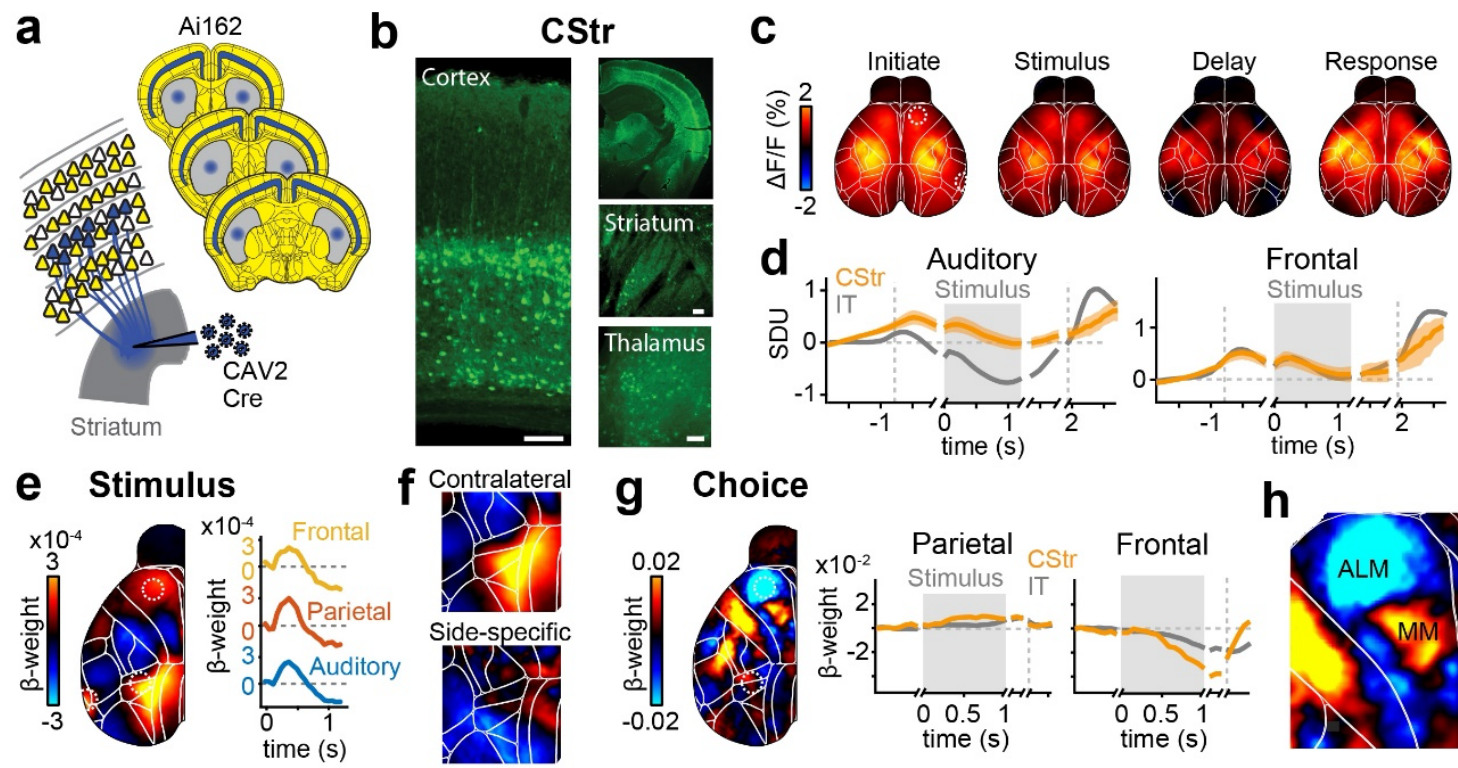

h
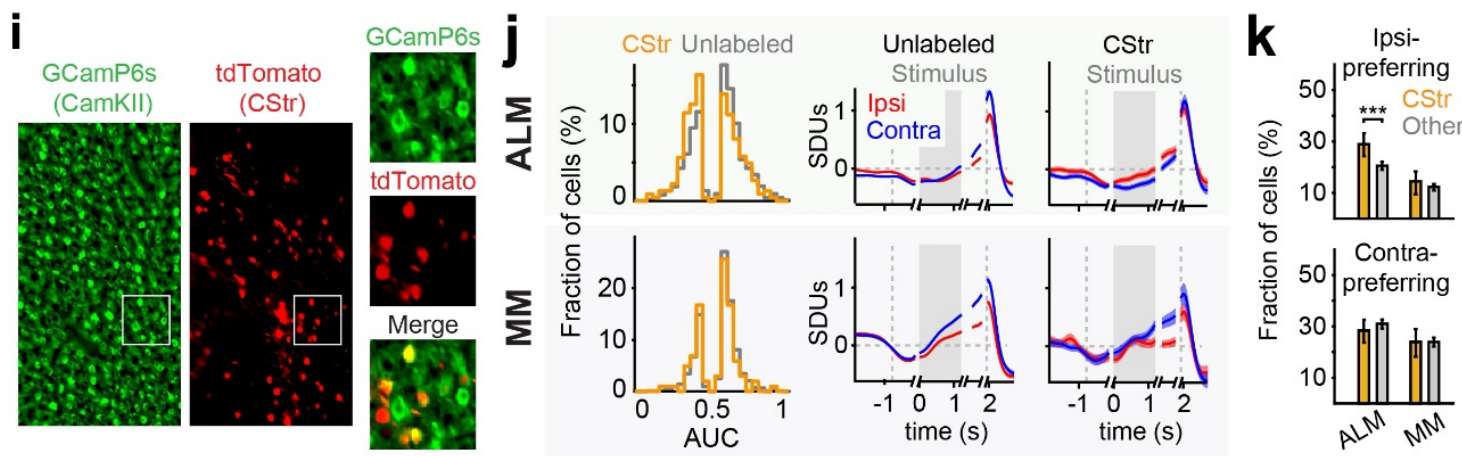

Figure 7. An intersectional approach to measure cortex-wide activity of CStr neurons.

a) Schematic of the retrograde labeling approach. Multiple bilateral injections of retrograde CAV2-Cre virus in Ai162 mice robustly induced expression of GCaMP6s in CStr neurons. b) GCaMP6s-expression was robust throughout the dorsal cortex, and largely confined to layer 5a. Scale bars are $100 \mu \mathrm{m}$. c) Cortical dynamics of CStr neurons in the auditory discrimination task. Shown are trial-averages over all correct, leftward trials in different trial episodes. No clear lateralization was observed in trial averages. d) Averaged activity in auditory (left) and frontal cortex (right) for CStr (orange) and IT (gray) mice. Dashed lines show times of initiation and response periods, gray areas the stimulus period. Traces show standard deviation units (SDU). e) Left: Contralateral stimulus kernel ("weight map"), averaged over all CStr mice, between 0 and $200 \mathrm{~ms}$ after stimulus onset. Right: Colored traces show changes in sensory (blue), parietal (red) and frontal cortex (yellow). Locations for each area are indicated by dashed circles in the weight map. f) Top: Weight map from (e), zoomed in on parietal cortex. Bottom: Difference of contra- versus ipsilateral stimulus kernels. g) Left: Cortical weight map from choice decoder during the delay period, averaged over all CStr mice. Right: Baseline-corrected decoder weights in parietal (left) and frontal cortex (right) for CStr (orange) and IT mice (gray). Traces are re-aligned to the initiation, stimulus, delay and response periods, indicated by gaps in weight traces. h) Weight map from (g), zoomed in on frontal cortex. i) Example fieldof-view from 2-photon imaging. Left panel: GCaMP6s-expression in all PyNs (green). Center panel: retrogradelylabeled CStr neurons, expressing tdTomato (red). Right panels: zoomed-in maps for both channels and a merged image. j) Left: Overview of significantly choice-tuned neurons in ALM (top) and MM (bottom). Orange line shows CStr neurons, gray lines are unlabeled PyNs. AUC values below 0.5 indicate stronger responses for ipsilateral choices. Right: Trial-averaged activity for all choice-selective neurons, separated for ipsi- (red) versus contralateral choices (blue). CStr neurons in ALM (top right) show higher activity for ipsilateral choices. k) Fraction of cells responding selectively for ipsi- (top) versus contralateral choices (bottom). A higher fraction of CStr neurons in ALM are ipsi-preferring (CStr: $28.9 \%$, Unlabeled: $20.5 \%$, binomial test: $p=5.9 \times 10^{-5}$ )

To confirm these results on a single-cell level, we used two-photon calcium imaging in Camk2 $\alpha$ tTA-G6s 2 mice and labeled CStr neurons through striatal injections of a retrograde adenoassociated virus (AAVrg-tdTomato) (Fig. 7i). This allowed us to compare the activity of all PyNs in frontal cortex with tdTomato-labeled CStr neurons in the same animal. Comparing the 
choice-tuning of CStr and unlabeled PyNs revealed a specific difference in ipsi- versus contralateral choice preference in ALM (Fig. 7j). Here, choice-selective CStr neurons preferentially responded to ipsilateral choices, whereas other PyNs were more contra-selective. Moreover, significantly more CStr neurons were tuned to ipsilateral choices than other PyNs (Fig. 7k). In agreement with our widefield results, these differences were only observed in ALM but not MM recordings.

The observed differences between PyN types suggest that these types may drive distinct aspects of decision-making. To causally test the functional role of different PyN types, we performed PyN-specific optogenetic inactivation. We induced Cre-dependent expression of the inhibitory opsin stGtACR2 ${ }^{44}$ by injecting an AAV in the parietal and frontal cortex of Fezf2-, PlexinD1-, or EMX-Cre mice (Fig. 8a, b). To express stGtACR2 in CStr neurons of wildtype mice, we combined cortical AAV injections with multiple CAV2-Cre injections in the striatum. Locations of parietal and frontal injections were based on our earlier stimulus and choice analyses (Fig. 5a, 6a; dashed circles). We placed glass fiber implants $(\varnothing=0.4 \mathrm{~mm})$ over each area to illuminate the cortical surface with blue light $\left(\lambda=470 \mathrm{~nm}\right.$, Irradiance $\left.=40 \mathrm{~mW} / \mathrm{mm}^{2}\right)$. To test whether optogenetic effects are area-specific, we also targeted the primary visual cortex (V1) in a subset of EMX mice. To simplify the behavioral task during the optogenetic inactivation experiments, we only presented auditory stimuli on one side in each trial and kept the duration of the stimulus and delay periods constant ( 1 and 0.5 seconds, respectively).

Bilateral optogenetic inactivation of either parietal or frontal cortex of EMX mice strongly impaired auditory task performance (Fig. 8c). Performance was unaffected by V1 inactivation, demonstrating that parietal and frontal cortex are selectively required for auditory decisions. We then illuminated each area for 0.5 seconds during four different task episodes: Early and late stimulus (the first and last 0.5 seconds of the stimulus period), delay and response (Fig. 8d). In agreement with our observation that parietal cortex reflects stimulus driven activity (Fig. 5a, red traces), parietal inactivation strongly reduced animal's performance, particularly during the early stimulus period (Fig. 8e). Behavioral impairments (computed as the normalized difference between performance in non-optogenetic trials and chance) were weaker during the subsequent task periods, indicating that parietal cortex is most important for early processing of auditory stimuli. As expected, impairments were strongest in EMX mice where all PyNs were affected (gray trace). Surprisingly, however, only inactivation of PT neurons resulted in a robust impairment, while behavioral effects were much weaker for IT or CStr neurons (Fig. 8e, blue versus green/orange traces). This suggests that the subcortical PT projection pathways in the parietal cortex have a larger causal impact on sensory processing than intracortical IT or CStr projections, pointing to a role for PT neurons that extends beyond preparation and execution of movements.

In frontal cortex, inactivation strongly impaired task performance in EMX mice, especially during the delay period, consistent with a role for frontal cortex in sensory integration and working memory (Fig. 8f, gray trace) ${ }^{20,41,45}$. IT and CStr inactivation resulted in comparable behavioral impairments during the stimulus and delay periods (green and orange traces). Choice impairments in IT mice are therefore not solely due to the disruption of intracortical processing ${ }^{20}$ but also involve alterations of CStr neurons. Inactivating PT neurons equally impaired animal performance during the stimulus and delay period but showed stronger effects during the final response period. Response impairments were similar for EMX and PT mice, suggesting that PT neurons are the main contributor to the execution of lick responses. These results show that multiple PyN types in frontal cortex are involved in the formation and maintenance of choices, despite clear differences in their respective tuning. However only PT neurons are required for subsequent motor execution. 

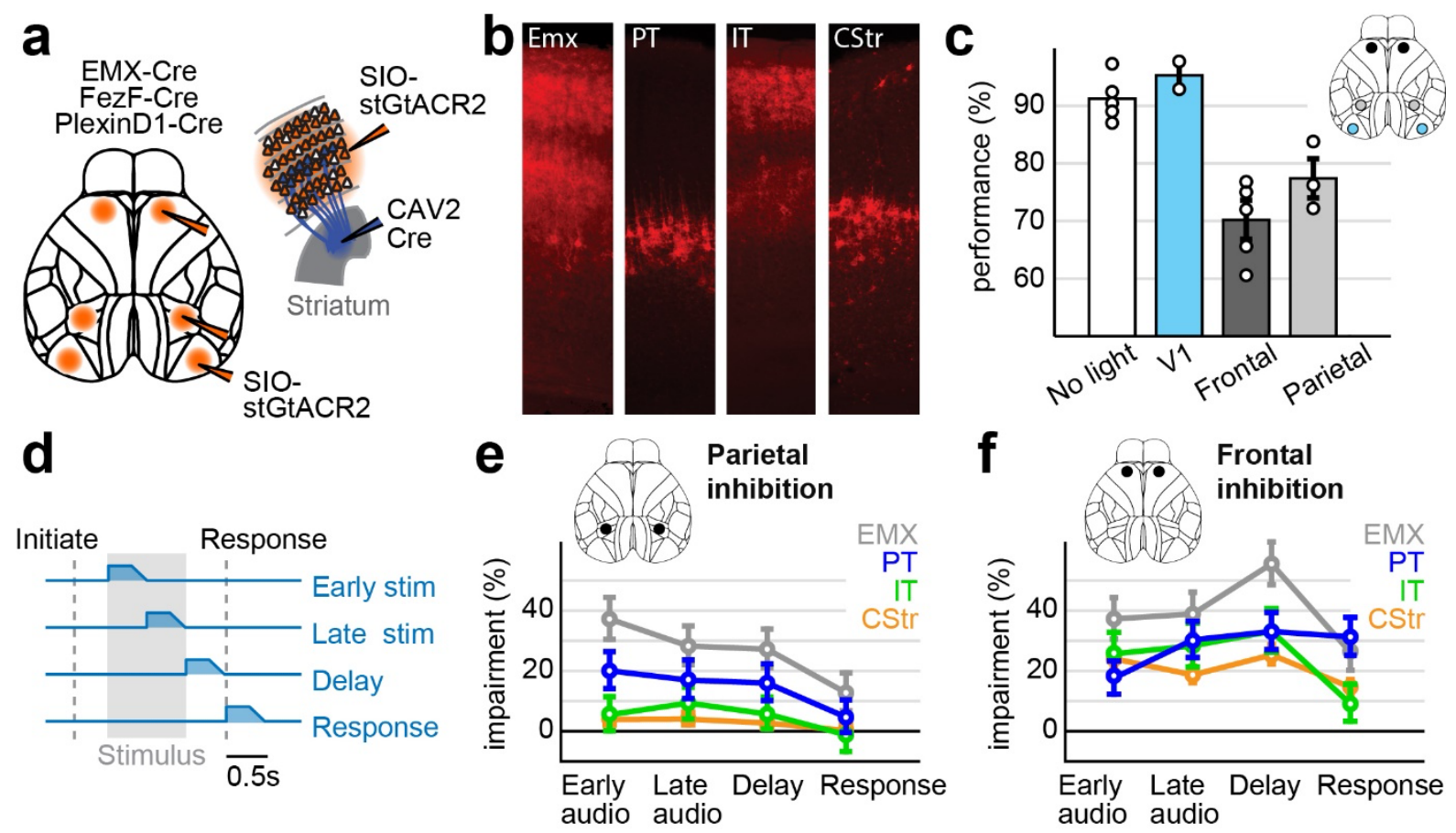

Figure 8. Temporally restricted, PyN-specific inactivation of parietal and frontal cortex disrupts decisions. a) Left: Schematic of injection scheme to induce stGtACR2 expression in EMX, IT or PT neurons. Injections were performed in frontal and parietal cortex of EMX-Cre, Fezf2-Cre or PlexinD1-Cre mice. V1 injections were performed in a subset of control EMX mice. Right: Retrograde CAV2-Cre virus injections in the striatum were combined with cortical injections of Cre-dependent stGtACR2 to selectively target CStr neurons. b) Layer-specific expression of stGtACR2-FusionRed in EMX, PT, IT and CStr neurons. c) Behavioral performance (\% correct) of EMX mice during inactivation of V1, frontal or parietal cortex. Both frontal and parietal inhibition reduced task performance. Circles indicate individual mice, error bars show the s.e.m. d) Schematic of optogenetic inactivation paradigm. 0.5-s long optogenetic inhibition was performed during the first or last part of the stimulus period, the subsequent delay or the response period. Light power ramped down after 0.3 seconds. e) Behavioral impairment (\% change from control performance) during inhibition of different PyN types in parietal cortex. Strongest effects are seen in EMX mice (gray) but also PT mice (blue). Impairments are weaker in IT (green) and CStr (orange) mice. Circles show mean impairments, error bars $95 \%$ confidence intervals. f) Behavioral impairment during inhibition of different PyN types in frontal cortex. Conventions as in e).

\section{Discussion}

We measured and manipulated the activity of specific PyN types to assess if they are largely functionally distinct or instead follow similar cortex-wide dynamics. Cortex-wide activity patterns were clearly PyN-type-specific, demonstrating that they reflect distinct neural dynamics at multiple spatial scales. PyN type specificity was also evident for functional signals during auditory decision-making: each PyN type exhibited unique stimulus- and choice-related response patterns with pronounced differences in cortical localization and spatial specificity. These response patterns were not observed when imaging from all PyNs unselectively. Optogenetic inactivation confirmed that PyN types in parietal and frontal cortex have distinct functional roles, highlighting the importance of subcortical projection pathways for sensory processing and choice formation. Taken together, our results suggest that different PyN types form parallel subnetworks throughout the cortex, are functionally-distinct, and perform separate roles during auditory decision-making.

In each PyN type, we observed unique cortex-wide activity patterns. By reducing cortical dynamics to a small set of spatiotemporal components ${ }^{33,46,47}$, we directly compared spatial activity patterns and found that virtually all spatial components were also PyN-type-specific. 
Differences between PyNs are therefore not only present with specific areas but are also evident in large-scale activity patterns. This has important implications for studies of cortex-wide neural dynamics, which are often based on indirect measures of neural activity, such as hemodynamic signals ${ }^{48,49}$, or pooled activity from all $\mathrm{PyNs}^{22,25,50,51}$. Earlier work revealed intricate circuit motifs and functional modules that span the entire cortex ${ }^{46,50-53}$ and largely follow intracortical connectivity patterns ${ }^{54,55}$. Our results strongly suggest that additional circuit motifs could be discovered when isolating the activity from different PyN types, especially subcortical projection types, such as PT or CStr neurons. Furthermore, most PyN-specific LocaNMF components consisted of spatially precise subregions that were smaller than Allen CCF areas. Detailed analysis of PyN-specific activity might therefore reveal more detailed structure than can be observed with nonspecific measures. For future studies, a particularly interesting approach to achieve this would be to combine large-scale measures of multiple PyN types with multi-color widefield imaging ${ }^{24,56}$. This would make it possible to directly observe interactions between PyN-specific cortical dynamics.

To isolate sensory activity, we used an encoding model and found clear responses in sensory, parietal and frontal cortex ${ }^{40,41,57,58}$. Consistent with our clustering results, these functional signals were unique for each PyN type and were either only partially represented in EMX mice or not observed at all. This functional segregation is in line with results from individual sensory areas, such as primary somatosensory ${ }^{18,59}$ and visual cortex ${ }^{19}$, and further suggests that different PyN types have separate roles during sensory processing. A recent study also found sensory responses in parietal cortex and established a causal role for the parietal area A in auditory decisionmaking ${ }^{58}$. In line with this, we also observed side-specific responses in area A for EMX and IT neurons. Surprisingly, sensory responses in PT and CStr neurons were located more medially and also differed in their side-specificity, suggesting that they might have distinct functional roles. To test this hypothesis, we performed PyN-specific parietal inactivation. Inactivation of parietal PT neurons during the stimulus presentation strongly disrupted eventual decisions. IT inactivation, by contrast, caused only weak behavioral effects. This argues against models in which task-relevant sensory information is directly transmitted from parietal to frontal cortex during decision formation ${ }^{40,58,60}$ and instead highlights the importance of subcortical projection pathways via PT neurons ${ }^{61-63}$. A likely subcortical target is the SC, which receives inputs from PT neurons ${ }^{18,26}$ and has been implicated with somatosensory ${ }^{18}$ and visual ${ }^{61,63}$ perception, and decision-making ${ }^{38,62,64}$. Parietal CStr neurons were not required for sensory perception and may thus serve a different function, such as tracking task history ${ }^{65}$.

Using a decoder analysis, we found that activity in the frontal cortex predicted the animal's left and right choices. This is consistent with earlier work ${ }^{20,40,45,66}$ but seems at odds with recent cortex-wide studies that found prominent movement-related activity but almost no choiceselectivity in cortex ${ }^{38,67}$. A potential reason could be that choice-related activity was indeed much weaker than movement signals and could therefore be obscured by movement signals if they are not separated in time ${ }^{37}$. Another possibility is that cortical circuits were more important for our specific task, because it included accumulation of sensory evidence and working memory ${ }^{67,68}$. This hypothesis is consistent with the ramping of choice signals we observed in PT neurons, which might reflect the activity of thalamus-projecting PT neurons during working memory ${ }^{13}$.

In contrast to other PyNs, CStr neurons were more selective for ipsilateral choices. Using twophoton imaging, we confirmed that this ipsilateral choice preference was also present for individual CStr neurons. This confirms that our widefield results indeed reflect somatic activity of CStr neurons and not just superficial dendritic signals, in line with earlier findings that widefield signals are strongly correlated to spiking of deeper cortical neurons ${ }^{42}$. Ipsilateral choice 
signals in CStr neurons were restricted to area ALM, which is mostly implicated in movement generation ${ }^{13,45}$. A recent study showed that CStr projections from the anterior cingulate cortex can inhibit striatal activity and motor behavior ${ }^{69}$. A potential reason for the reduced activity of CStr neurons during the response period could therefore be to disinhibit striatal circuits and release a targeted licking response.

In agreement with our imaging results, frontal inactivation strongly impaired animal behavior during the stimulus and delay period, suggesting an important role for the translation of sensory inputs into behavior ${ }^{21,67,70-72}$. Impairments were largely similar for all frontal PyN types, which appear to be equally required for choice formation and retention. Frontal PyNs may thus be more reliant on each other to maintain accurate function than in sensory areas ${ }^{18,19,73}$. As the only exception, PT neurons were more important during the response period, consistent with a specific role of brainstem-projecting PT neurons for motor execution ${ }^{13}$.

Our PyN-specific imaging largely relied on mice where GCaMP-expression was restricted to specific neural subpopulations. A possible concern is hereby the extent to which different PyNtypes might still be mixed together. Indeed, many layer-specific mouse lines often combine PyNtypes with diverse projection targets ${ }^{13,17,58,59}$. Moreover, transgenes in most PyN driver lines are inserted at random integration sites, which can lead to unstable expression patterns across cortical areas or mixtures of cell types due to interactions with surrounding genetic elements ${ }^{26,74}$. Our temporally inducible knock-in lines target developmental markers and closely reflect endogenous gene expression during brain development, resulting in more even and specific expression in cortex ${ }^{26}$. Focusing on PT and IT neurons, also allowed us target two well-separated major PyN classes ${ }^{17}$ with known functional differences in some areas ${ }^{18,19}$. Future studies could explore PyN type function at even higher granularity by using more specific mouse lines, such as the Cux1-line which isolates intracortically-projecting IT neurons ${ }^{26}$. Another way to separate PyN types with different projection targets, such as IT neurons that project to cortex and striatum, is retrograde labeling. Our results show that this approach can be leveraged to monitor the cortex-wide activity of a PyN type based on projection target alone. Since the appropriate definition of a PyN type may depend on the question at hand, both the genetic and retrograde approaches will be useful for future studies that aim to study cortex-wide activity of PyN-types in different behavioral contexts.

Our work offers a new perspective on cortex-wide dynamics by viewing them through the lens of different PyN types and strongly supports the view that cortical circuits perform parallel computations, even within the same cortical layer ${ }^{13,18,19,75}$. We defined cell types either by developmental lineage or projection target because a unifying definition remains elusive. Subcortical areas are targeted by genetically-distinct PyNs ${ }^{12,26}$ and broader PyN types, such as PT neurons, contain subtypes with different projection targets ${ }^{13}$. Future studies may therefore combine retrograde labeling and transgenic lines to target PyN subtypes with even higher specificity ${ }^{26}$ and resolve the functional heterogeneity that is often encountered when studying cortical decision circuits without the right tools.

\section{Acknowledgements}

We thank Z. Josh Huang for providing us with Fezf2 and PlexinD1 mice, as well as for many helpful conversations. We thank Shreya Saxena and Joao Cuoto for technical advice and scientific discussions and Anup Khanal for helping with perfusions. We thank Adam Kepecs and Shujing Li for providing us with the AAV-DJ-hCAR virus. Financial support was received from the Deutsche Forschungsgemeinschaft (SM, German Research Foundation, DFG 368482240/GRK2416), NIH MH120888 (XRS). 


\section{Methods}

\section{Mouse lines}

All surgical and behavioral procedures conformed to the guidelines established by the National Institutes of Health and were approved by the Institutional Animal Care and Use Committee of Cold Spring Harbor Laboratory. Experiments were conducted with male mice between the ages of 8 to 25 weeks. No statistical methods were used to pre-determine sample sizes but our sample sizes are similar to those reported in previous publications ${ }^{22,25}$. All mouse strains were acquired from the Jackson Laboratory, the Allen Brain Institute, or generated at Cold Spring Harbor Laboratory. Transgenic strains crossed to generate double- and triple-transgenic mice used for imaging: EmxCre (JAX 005628), LSL-tTA (JAX 008600), Ai93D (JAX 024103), Ai162 (JAX 031562), G6s2 (JAX 024742) and H2B-eGFP (JAX 006069). EMX mice, used for calcium imaging, were bred as Ai93D;Emx-Cre;LSL-tTA. To avoid potential aberrant cortical activity patterns, EMX mice were on a doxycycline-containing diet (DOX), preventing GCaMP6 expression until they were 6 weeks or older ${ }^{22,25}$. To obtain PT- and IT-specific transgenic lines, we used two inducible knock-in mouse lines (Fezf2-2A-CreER and PlexinD1-2A-CreER) that were generated by inserting a 2A-CreER or 2A-Flp cassette in-frame before the STOP codon of the targeted gene. Both strains have been extensively characterized to reflect endogenous gene expression patterns that are closely linked to specific excitatory neuron types and induce robust and uniform expression throughout the cortex ${ }^{26}$. They are available from Jackson Laboratory (Fezf2-2A-CreER, JAX 036296 and PlexinD1-2ACreER, JAX 036295). For widefield imaging, each strain was mated with Ai162 reporter mice to induce expression of GCaMP6s in Fezf2- or PlexinD1-positive neurons. Additionally, we crossed Ai162 with G6s2 (tetO-GCaMP6s, JAX 024742) to create the hybrid reporter strain Ai162;G6s2, with Cre-dependent tTA expression as well as two copies of GCaMP6s under tetO control. This hybrid reporter line is not affected by leaky reporter gene expression seen in Ai162 homozygotes and expresses GCaMP6s at higher levels than Ai162 hemizygotes. To express GCaMP6s for widefield imaging in corticostriatal projection neurons, we performed striatal injections of retrograde viral vector using the hybrid Ai162;G6s2 reporter line (see section: viral injections).

\section{General surgical procedures}

All surgeries were performed under 1-2\% isoflurane in oxygen anesthesia. After induction of anesthesia, $1.2 \mathrm{mg} / \mathrm{kg}$ of meloxicam was injected subcutaneously and sterile lidocaine ointment was applied topically to the skin incision site. After making a midline cranial incision, the skin was retracted laterally and fixed in position with tissue adhesive (Vetbond, 3M). We then built an outer wall using dental cement (C\&B Metabond, Parkell; Ortho-Jet, Lang Dental) along the lateral edge of the dorsal cranium (frontal and parietal bones) to maximize the area of exposed skull. A custom titanium skull post was then attached to the dental cement. For skull clearing, debris and periosteum were thoroughly cleaned from the skull followed by the application of a thin layer of cyanoacrylate (Zap-A-Gap CA+, Pacer technology).

To prepare mice for two-photon imaging, a circular craniotomy $(\varnothing=3 \mathrm{~mm})$ centered over the right frontal cortex (1.75 mm lateral to midline and $1.75 \mathrm{~mm}$ rostral to bregma), was made using a biopsy punch. A circular coverslip $(\varnothing=3 \mathrm{~mm})$ was then lowered to the surface of the brain and Vetbond and Metabond were used to seal the window. Lastly, a titanium skull post was implanted as described above. 


\section{Viral injections}

After induction with isoflurane anesthesia, animals were placed in a stereotaxic frame (David Kopf Instruments). The skull was leveled along both the rostral-caudal and medio-lateral axis, allowing precise and reproducible targeting. All injections were made using a programmable nanoliter injector (Nanoject III, Drummond Scientific, PA). Retrograde targeting of pan-corticostriatal projection neurons were performed in C57BL/6J mice (3-4 weeks of age) through bilateral stereotaxic injections of CAV-2-Cre into the dorsal striatum at three striatal targets per hemisphere spanning the rostro-caudal axis. The coordinates relative to bregma (in mm) are (1) $\mathrm{RC}+0.75, \mathrm{ML}$ \pm 1.8 , DV 3.0; (2) RC 0, ML \pm 2.2 , DV 3.1; (3) RC -0.75, ML \pm 2.9 , DV 3.1. For each striatal target, a minimal craniotomy was created using a small dental burr followed by injection of $1.8 \times 109$ purified particles (pp) of CAV-2-Cre using pipettes with long taper tips pulled from borosilicate capillaries (3.5" Drummond \# 3-000-203-G/X, Drummond Scientific, PA). To retrogradely label CStr neurons during two-photon imaging we injected AAVretro-tdTomato, using the same approach and coordinates as described above.

For cell type-specific optogenetic silencing experiments, we performed bilateral frontal and parietal cortex injections (coordinates relative to bregma (in mm): frontal cortex: RC 2.5, ML \pm 1.5 ; parietal cortex: RC -1.7, ML \pm 2.5 ) of Cre-dependent stGtACR2 (AAV1-hSyn-SIO-stGtACR2FusionRed, Upenn Vector Core). Injections were made in Fezf2-2A-CreER, PlexinD1-2A-CreER, and EMX-Cre (6-8 weeks old) reporter mice to induce expression of stGtACR2 in different PyN types. In mice with ligand-activated Cre recombinase activity, intraperitoneal tamoxifen was administered one week after viral injections. Injections $(4 \times 108 \mathrm{vg}$ per injection) were made at two depths $(300$ and $600 \mu \mathrm{m})$ per cortical target. In two EMX-Cre mice, bilateral injections were performed in the frontal and visual cortex ( $\mathrm{RC}-4, \mathrm{ML} \pm 2.5)$. To express stGtACR2 in corticostriatal projection neurons, $\mathrm{C} 57 \mathrm{BL} / 6 \mathrm{~J}$ mice were injected in two stages. A mixture of AAV1-SIO-hSyn1-stGtACR2-FusionRed and AAV-DJ-hCAR (a gift from the laboratory of Adam Kepecs) was injected into the cortex of 3-4-week-old mice, followed by bilateral striatal CAV-2-Cre injections 6 weeks later (as described above).

\section{Optical fiber implantation}

For optogenetic silencing, we used the soma-targeted anion-conducting channelrhodopsin stGtACR2 in target neuronal populations $^{44}$. Optical fibers (NA $=0.36, \varnothing=0.4 \mathrm{~mm}$, FT400UMT, Thorlabs) were glued into metal or ceramic ferrules $(\varnothing=1.25 \mathrm{~mm}$, Thorlabs) and secured above the cortex following viral injections at each target. In Fezf2 and PlexinD1 mice, ferrule-enclosed fibers were implanted immediately following cortical AAV injection. In CStr mice, fiber implantation immediately followed striatal CAV-2-Cre injections. Optical fibers were implanted extradural to the site of cortical injections and interfaced with a thinned layer of skull using cyanoacrylate. Upon curing of the cyanoacrylate, the fiber was secured to the skull using lightcured glass ionomer (Vitrebond, 3M). Additional layers of dental cement as well as dental acrylic (Lang Dental Jet Repair Acrylic; Part\#1223MEH) were applied to reinforce the implants for durability and long-term stability. After all layers were cured, an outer coating of cyanoacrylate as well as nail polish were applied.

\section{Behavioral training}

The behavioral setup was controlled with a microcontroller-based (Arduino Due) finite state machine (Bpod r0.5, Sanworks) using custom Matlab code (2015b, Mathworks) running on a Linux PC. Servo motors (Turnigy TGY-306G-HV) and touch sensors were controlled by microcontrollers (Teensy 3.2, PJRC) running custom code. Forty-five mice were trained on a 
delayed, spatial discrimination task. Mice initiated trials by placing their forepaws on at least one of the two handles, which were mounted on servo motors that rotated out of reach during the intertrial period. Upon trial initiation, animals placed their forepaws on the handles and, after a variable duration of $0.25-0.75$ seconds of continuous contact, the auditory stimulus was presented. Auditory stimuli consisted of a sequence of Poisson-distributed, 3-ms long auditory click sounds ${ }^{35}$, presented from either a left and/or right speaker for a variable duration between 1 and 1.5 seconds. The stimulus period was followed by a variable delay of up to 1 second, then the servo motors moved two lick spouts into close proximity of the animal's mouth. If the animal licked twice on the side where more clicks were presented, a drop of water reward was dispensed. The amount of water rewarded per trial (typically 1.5 to $3 \mu \mathrm{l}$ ) is constant within a single session but may be adjusted daily based on the animal's body weight. After one spout has been licked twice, the contralateral spout moved out of reach to force the animal to commit to its decision.

All trained mice were housed in groups of two or more under reverse light cycle (12- hour dark and 12-hour light) and trained during their active dark cycle. Animals were trained over the course of approximately 30-60 days. After 2-3 days of restricted water access, animals began habituation to head fixation and received water from spouts in the behavior chamber. During these sessions, unilateral auditory stimuli were presented followed by a droplet of water dispensed freely from the ipsilateral water spout. After several habituation sessions, animals were then required to touch the handles to trigger stimulus presentation. Once mice could reliably reach for the handles, the required touch duration was progressively increased up to 0.75 seconds. During the next stage of training, self-performed trials, where both spouts moved within reach of the animal following stimulus presentation, were progressively introduced. An animal was considered trained when its detection performance across two or more sessions was above $80 \%$.

\section{Behavioral monitoring}

Data was collected from multiple sensors in the behavioral setup to measure different aspects of animal movement. Touch sensors using a grounding circuit on handles and lick spouts detected contact with the animal's forepaws and tongue, respectively. A piezo sensor (1740, Adafruit LLC) below the animal's trunk was used for monitoring body and hindlimb movements. Two webcams (C920 and B920, Logitech) were used for video recording of animal movements. Cameras were positioned to capture the animal's face (side view) and the ventral surface of the body (ventral view).

\section{Widefield imaging}

Widefield imaging was done as reported previously ${ }^{23,32,76}$ using an inverted tandem-lens macroscope in combination with an sCMOS camera (Edge 5.5, PCO) running at 30 fps. The top lens had a focal length of $105 \mathrm{~mm}$ (DC-Nikkor, Nikon) and the bottom lens $85 \mathrm{~mm}$ (85M-S, Rokinon). The field of view was $12.5 \times 10.5 \mathrm{~mm}^{2}$ and the imaging resolution was $640 \times 540$ pixels after 4x spatial binning, resulting in a spatial resolution of $\sim 20 \mu \mathrm{m}$ per pixel. To capture GCaMP fluorescence, a $525 \mathrm{~nm}$ band-pass filter (\#86-963, Edmund optics) was placed in front of the camera. Using excitation light at two different wavelengths, we isolated $\mathrm{Ca}^{2+}$-dependent fluorescence and corrected for intrinsic signals (e.g., hemodynamic responses) ${ }^{22,25}$. Excitation light was projected on the cortical surface using a $495 \mathrm{~nm}$ long-pass dichroic mirror (T4951pxr, Chroma) placed between the two macro lenses. The excitation light was generated by a collimated blue LED (470 nm, M470L3, Thorlabs) and a collimated violet LED (405 nm, M405L3, Thorlabs) that were coupled into the same excitation path using a dichroic mirror (\#87-063, Edmund optics). We alternated illumination between the two LEDs from frame to frame, resulting in one set of frames with blue and the other with violet excitation at $15 \mathrm{fps}$ each. Excitation of GCaMP at $405 \mathrm{~nm}$ 
results in non-calcium dependent fluorescence ${ }^{77}$, allowing us to isolate the true calcium-dependent signal by rescaling and subtracting frames with violet illumination from the preceding frames with blue illumination. All subsequent analysis was based on this differential signal. The imaging data was then rigidly aligned to the Allen common coordinate framework (CCF), using four anatomical landmarks: the left, center and right points where anterior cortex meets the olfactory bulbs, and the medial point at the base of retrosplenial cortex. Retinotopic visual mapping experiments ${ }^{31,78}$ confirmed accurate $\mathrm{CCF}$ alignment and showed high correspondence between functionally identified visual areas and the CCF across PyN types (Fig. 1c).

\section{Two-photon imaging}

We used a two-photon resonant scanning microscope at 30.9 frames per second (Moveable Objective Microscope, Sutter Instruments). A 16X, 0.8 NA Nikon objective lens was used for single-plane imaging with a $512 \times 512$ pixels $(575 \mu \mathrm{m}$ x $575 \mu \mathrm{m})$ fields of view. Mode-locked illumination at $930 \mathrm{~nm}$ was delivered using a Ti:Sapphire laser (Ultra II, Coherent). Images were acquired continuously at $30.9 \mathrm{~Hz}$. Two-photon frames and behavior control events were aligned through synchronized acquisition of analog galvanometric and Bpod signals. Depth of focal planes was $200-400 \mu \mathrm{m}$ below the pial surface. Emission was collected using band-pass red $(670 / 50 \mathrm{~nm})$ and green $(525 / 50 \mathrm{~nm})$ filters (Chroma Technologies). MScan software (Sutter Instruments) was used for image acquisition. Recordings were either done in ALM (1.5 mm lateral and $2.5 \mathrm{~mm}$ anterior to bregma) and MM (1 $\mathrm{mm}$ lateral and $1.5 \mathrm{~mm}$ anterior to bregma) in randomized order across mice. Across imaging session, we avoided imaging previously acquired planes to maximize the number of unique neurons by selecting planes that differed from those of prior sessions.

\section{Optogenetic inactivation}

Photostimulation was performed using light from a $470 \mathrm{~nm}$ high-power LED (M470F3, Thorlabs) with a power density of $10 \mathrm{~mW} / \mathrm{mm}^{2}$. Stimuli consisted of a square wave stimulus that ramped down in power for 200 milliseconds, to avoid an excitatory post-illumination rebound due to sudden release of inhibition ${ }^{79}$. To prevent animals' visual detection of photostimulation, either through external leakage from light-insulated mating sleeves or transmission to the retina across the brain, an external LED with matching wavelength placed at the center of the animal's visual field was flashed throughout the duration of every trial. Photoinhibition was performed in $20 \%$ of total trials and randomly interleaved between light-off trials. During each session, only bilateral frontal, parietal or visual cortex inhibition was performed. Once an animal was habituated and able to complete detection behavior trials with $>90 \%$ accuracy, optogenetic inactivation trials were introduced. During these initial sessions, optogenetic inhibition was performed from the beginning of the stimulus epoch until the end of the delay epoch. Additionally, we performed 0.5 -second inhibition during four pre-defined epochs of the detection behavior trials: (1) first half of the stimulus, (2) second half of the stimulus, (3) delay, (4) response.

\section{Immunohistology, microscopy and image analysis}

For a given animal, after all experiments were concluded, we performed transcardial perfusion with PBS followed by fixation with 4\% PFA in $0.1 \mathrm{M} \mathrm{PB}$. Brains were post-fixed in 4\% PFA for an additional $12-18$ hours at $4^{\circ}$. Prior to sectioning, brains were rinsed three times in PBS and embedded in $4 \%$ agarose-PBS. Slices $50 \mu \mathrm{m}$ in thickness were made using a vibrating microtome (Leica, VT100S). Sections were then suspended in blocking solution (10\% Normal Goat Serum and $0.1 \%$ Triton-X100 in $1 \mathrm{X}$ PBS) for 1 hour followed by overnight incubation at $4{ }^{\circ} \mathrm{C}$ with the primary antibody. Next, sections were washed with PBS, incubated for $1 \mathrm{~h}$ at room temperature with the secondary antibody at 1:500 dilution. For visualization of GCaMP6s, we used primary 
goat polyclonal anti-GFP antibody (Abcam) and secondary donkey anti-goat Alexa Fluor 488 (Abcam). Sections were then dry-mounted on slides using Vectashield (Vector Labs, H1000) prior to imaging. Imaging was performed using upright fluorescence macroscope and microscope (Olympus BX61). Images were acquired using Ocular Scientific Image Acquisition Software (Teledyne Imaging) and visualization and analysis were performed using ImageJ/FIJI software packages.

\section{Preprocessing of neural data}

We first performed motion correction on each imaging frame, using a rigid-body image registration method implemented in the frequency domain ${ }^{80}$ that aligned each frame to the median over all frames in the first trial. To reduce the computational cost of subsequent analyses, we then computed the 200 highest-variance components using singular value decomposition (SVD). These components accounted for at least $95 \%$ of the total variance in each recording, whereas computing 500 components accounted for little additional variance $(\sim 0.15 \%)$. SVD reduces the raw imaging data $\mathrm{Y}$ to a matrix of 'spatial components' $\mathrm{U}$ (of size pixels by components), 'temporal components' $\mathrm{V}^{\mathrm{T}}$ (of size components by frames) and singular values $\mathrm{S}$ (of size components by components) to scale temporal components to the original data. The resulting decomposition has the form $\mathrm{Y}=\mathrm{USV}^{\mathrm{T}}$. All subsequent analysis in the time domain (such as the encoder and decoder models described below) were then performed on the product $\mathrm{SV}^{\mathrm{T}}$ and the respective results were later multiplied with $U$, to recover results for the original pixel space. To avoid slow drift in the imaging data, SVT was high-pass filtered above $0.1 \mathrm{~Hz}$ using a zero-phase, second-order Butterworth filter.

To compute trial averages and perform choice decoder analysis (see below), imaging data in individual trials were aligned to the four trial periods, each marked by a specific event. This was required because the duration of different trial events was randomized to reduce temporal correlations, e.g. between trial initiation, the stimulus presentation and subsequent lick responses. The first period (Initiate) was aligned to the time when animal initiated a trial by touching the handles, the second (Stimulus) was aligned to the stimulus onset, the third (Delay) to the end of the stimulus sequence, and the fourth (Response) to the time when spouts were moved in to allow a lick response. After alignment, the total trial duration was 2 seconds and durations of respective trial episodes were 0.5 (Initiate), 1 (Stimulus), 0.2 (Delay), and 0.3 seconds (Response).

\section{Spatial clustering and classification}

To obtain more interpretable spatial components and assess the dimensionality of cortical activity in different PyN types, we used semi-nonnegative matrix factorization (sNMF). As with SVD, sNMF also creates spatial and temporal components for each session and for each mouse but enforces that spatial components are strictly positive. We used the LocaNMF toolbox by Saxena et $\mathrm{al}^{33}$ (https://github.com/ikinsella/locaNMF) to transform the spatial and temporal components $\mathrm{U}$ and $\mathrm{SV}^{\mathrm{T}}$ into two corresponding matrices $\mathrm{A}$ and $\mathrm{C}$, where $\mathrm{A}$ is a matrix of non-negative spatial components (of size pixels by components) and $\mathrm{C}$ the corresponding temporal components (of size components by frames). In addition to regular sNMF, the LocaNMF toolbox can be initialized with spatial constraints that are based on the Allen CCF. To obtain spatially restricted localized LocaNMF components, we constructed a map of larger seed regions by merging several smaller areas in the Allen CCF together (Fig. 2e). This region map is then used to enforce that each component in A is sparse outside the boundary of a given region. The amount of possible overlap between regions is specified by a localization threshold which specifies the percentage of a given component that is constrained to be inside a single region's boundary. To obtain dense spatial components that were mostly driven by the local correlations between pixels and could also lie at 
the border between seed regions, we used a localization threshold of 50\%. To obtain unconstrained sNMF spatial components, we also used the LocaNMF toolbox but only provided a single region that spanned the entire cortex. This resulted in cortex-wide components, similar to vanilla sNMF, while ensuring that all other analysis steps were done identically for sNMF and LocaNMF components. In both cases, we determined how many components in $\mathrm{A}$ and $\mathrm{C}$ were needed to explain $99 \%$ of the variance of $Y$ (with $Y=A C$ ) after the initial SVD.

To compare spatial sNMF and LocaNMF components from different PyN types, we embedded them in a 2-dimensional space, using Uniform Manifold Approximation and Projection (UMAP) analysis (Fig. 2c,g). UMAP analysis was performed with the UMAP toolbox by McInnes et al. ${ }^{34}$ (https://github.com/lmcinnes/umap). For each recording, the first 20 spatial components in A (either from sNMF or region-constrained LocaNMF) were downsampled by a factor of 2 , smoothed with a 2-D gaussian filter ( 5 x 5 pixels, 2 pixel standard deviation) and peak-normalized. Components from all recordings and animals were then combined into a larger matrix (of size pixels by components) and we used UMAP to project the first (pixel) dimension into two, maximally separating non-linear dimensions. Each point in the two dimensional space (Fig. 2c,g) therefore reflects a single component from one animal in a given imaging session.

To identify PyN types based on individual components, we tested each of the first 20 components in each session 10 times (200 repetitions per session). In every repetition, 500 components were randomly selected from all mice (except the one from which the current component was taken) and we assigned the PyN type based on the majority type in the 10 nearest neighbors of the current component in UMAP space. Classifier accuracy for each session (Fig. 2d,h) was then computed as the mean probability over all repetitions to accurately identify the PyN type. The same procedure was used for both sNMF or locaNMF components.

\section{Linear encoding model}

The linear encoding model was based on a combination of task- and movement-related variables, as described previously ${ }^{32}$. Each variable consisted of multiple regressors that were combined into a larger design matrix. Binary regressors contained a single pulse that signaled the occurrence of specific events, such as the stimulus onset, and additional regression copies that were shifted forward or backward in time to account for changes in cortical activity before or after the respective event. For auditory stimuli, the time-shifted copies spanned all frames from the onset of the auditory sequence until the end of the trial. Individual click sounds were also captured by an additional regressor set that spanned the 2 seconds after click onset. For motor events, like licking or whisking, the time-shifted copies spanned the frames from $1 \mathrm{~s}$ before until $2 \mathrm{~s}$ after each event. Lastly, for some variables, such as the previous choice, the time-shifted copies spanned the whole trial. Other variables were analog, such as measures from the piezo sensor or the pupil diameter, and also contained the 200 highest temporal components of video information from both cameras (using SVD as described above). This ensured that the model could account for animal movements and accurately isolate task-related activity. Movement and task variables were additionally decorrelated due to the variable durations of the initiation, stimulus and delay period. The model was fit using ridge regression and the regularization penalty was estimated separately for each column of the widefield data using marginal maximum likelihood estimation ${ }^{81}$. Ridge regression was chosen to allow for similar contributions from different correlated variables.

\section{Variance analysis}

Explained variance $\left(\mathrm{cvR}^{2}\right)$ was obtained using 10-fold cross-validation. To assess unique explained variance by individual variables $\left(\Delta \mathrm{R}^{2}\right)$, we created reduced models in which all 
regressors of a specific variable were shuffled in time. The difference in explained variance between the full and the reduced model yielded the unique contribution $\Delta \mathrm{R}^{2}$ of that model variable that could not be explained by other variables in the model. The same approach was used to compute unique contributions for groups of variables, i.e., 'Movements' and 'Task'. Here, all variables that corresponded to a given group were shuffled at once.

\section{Decoding model}

To predict animal's left/right choices from widefield data, we trained logistic regression decoders with an L1 penalty on the temporal component matrix SV $\mathrm{SV}^{\mathrm{T}}$ in each session. When decoding choice, we randomly removed trials until there was an equal amount of correct and incorrect trials where mice chose the left and the right side. By balancing left/right choice sides and correct/incorrect trials, we ensured that the decoder would not predict choices due to corresponding sensory information or would be influenced by potential side biases. The logistic regression model was implemented in Matlab using the 'fitclinear' function and run repeatedly for each time point in individual trials after re-aligning them to trial periods as described above. In each session, all decoder runs were performed with the same amount of trials (at least 250 trials) and we used 10fold cross-validation to compute decoder accuracy at each time point in the trial. Beta weights were averaged from all models created during cross-validation and convolved with the spatial component matrix $\mathrm{U}$ to create cortical maps of decoder weights. 


\begin{tabular}{|l|l|}
\hline Optogenetics \\
\hline Cell type & Number of mice \\
\hline Emx & 5 \\
\hline CStr & 5 \\
\hline Fezf2 & 8 \\
\hline PlexinD1 & 8 \\
\hline Widefield & \\
\hline Emx & 4 \\
\hline CStr & 6 \\
\hline Fezf2 & 5 \\
\hline PlexinD1 & 4 \\
\hline Two-photon & \\
\hline CStr & 3 \\
\hline
\end{tabular}

\begin{tabular}{|c|c|c|}
\hline Reagent/resource & Source & Identifier \\
\hline \multicolumn{3}{|l|}{ Antibodies } \\
\hline Goat polyclonal anti-GFP & Abcam & ab6673 \\
\hline Donkey Anti-Goat Alexa Fluor 488 & Abcam & ab150129 \\
\hline \multicolumn{3}{|l|}{ Viral strains } \\
\hline CAV-2-Cre & $\begin{array}{l}\text { Plateforme de } \\
\text { Vectorologie de } \\
\text { Montpellier }\end{array}$ & N/A \\
\hline AAV1-hSyn1-SIO-stGtACR2-FusionRed & Penn Vector Core & $\begin{array}{l}105677- \\
\text { AAV1 }\end{array}$ \\
\hline AAVrg-CAG-tdTomato & Penn Vector Core & $\begin{array}{l}59462- \\
\text { AAVrg }\end{array}$ \\
\hline AAV-DJ-hCAR & $\begin{array}{l}\text { Laboratory of Adam } \\
\text { Kepecs }\end{array}$ & \\
\hline \multicolumn{3}{|l|}{ Experimental Models } \\
\hline Mouse: Emx1-IRES-Cre: Emx1 ${ }^{\text {tm1(cre)Krj }}$ & The Jackson Laboratory & JAX\#005628 \\
\hline $\begin{array}{l}\text { Mouse: ROSA:LNL:tTA: } \\
\text { Gt(ROSA)26Sor }{ }^{\text {tm1(tTA)Roos }}\end{array}$ & The Jackson Laboratory & JAX\#008600 \\
\hline Mouse: Camk2 $\alpha$-tTA: Tg(Camk2a-tTA)1Mmay & The Jackson Laboratory & JAX\#003010 \\
\hline $\begin{array}{l}\text { Mouse: Ai93(TITL-GCaMP6f)-D (Ai93D): } \\
\text { Igs } 7^{\text {tm93.1(tetO-GCaMP6f)Hze }}\end{array}$ & The Jackson Laboratory & JAX\#024103 \\
\hline $\begin{array}{l}\text { Mouse: Ai162(TIT2L-GC6s-ICL-tTA2)-D } \\
\text { (Ai162D): Igs } 7^{\text {tm162.1(tetO-GCaMP6s,CAG-tTA2)Hze }}\end{array}$ & $\begin{array}{l}\text { H. Zeng, Allen Institute } \\
\text { for Brain Science }\end{array}$ & JAX\#031562 \\
\hline $\begin{array}{l}\text { Mouse: TRE-GCaMP6s (G6s2): Tg(tetO- } \\
\text { GCaMP6s)2Niell }\end{array}$ & The Jackson Laboratory & JAX\#024742 \\
\hline Mouse: H2B-eGFP: Tg(HIST1H2BB/EGFP)1Pa & The Jackson Laboratory & JAX\#006069 \\
\hline Mouse: Fezf2-2A-CreER: Fezf2 ${ }^{\text {tm1.1(cre/ERT2)Zjh }}$ & The Jackson Laboratory & JAX\#036296 \\
\hline Mouse: PlexinD1-2A-CreER: Plxnd1 ${ }^{\text {tm2.1(flpo)Zjh }}$ & The Jackson Laboratory & $\mathrm{JAX} \# 036295$ \\
\hline \multicolumn{3}{|l|}{ Software } \\
\hline MATLAB 2018B & Mathworks & \\
\hline Python 3.6.10 & $\begin{array}{l}\text { Python Software } \\
\text { Foundation }\end{array}$ & \\
\hline \multicolumn{3}{|l|}{ Other } \\
\hline Bpod State Machine r0.5 & Sanworks & $\mathrm{N} / \mathrm{A}$ \\
\hline
\end{tabular}




\section{References}

1. Harris, K. D. \& Mrsic-Flogel, T. D. Cortical connectivity and sensory coding. Nature 503, 51-58 (2013).

2. Pfeffer, C. K., Xue, M., He, M., Huang, Z. J. \& Scanziani, M. Inhibition of Inhibition in Visual Cortex: The Logic of Connections Between Molecularly Distinct Interneurons. Nat Neurosci 16, 1068-1076 (2013).

3. Keller, A. J. et al. A Disinhibitory Circuit for Contextual Modulation in Primary Visual Cortex. Neuron 108, 1181-1193.e8 (2020).

4. Tremblay, R., Lee, S. \& Rudy, B. GABAergic Interneurons in the Neocortex: From Cellular Properties to Circuits. Neuron 91, 260-292 (2016).

5. Cardin, J. A. et al. Driving fast-spiking cells induces gamma rhythm and controls sensory responses. Nature 459, 663-667 (2009).

6. Veit, J., Hakim, R., Jadi, M. P., Sejnowski, T. J. \& Adesnik, H. Cortical gamma band synchronization through somatostatin interneurons. Nature Neuroscience 20, 951-959 (2017).

7. Sohal, V. S., Zhang, F., Yizhar, O. \& Deisseroth, K. Parvalbumin neurons and gamma rhythms enhance cortical circuit performance. Nature 459, 698-702 (2009).

8. Reimer, J. et al. Pupil fluctuations track fast switching of cortical states during quiet wakefulness. Neuron 84, 355-362 (2014).

9. Fu, Y. et al. A Cortical Circuit for Gain Control by Behavioral State. Cell 156, 1139-1152 (2014).

10. Zhou, M. et al. Scaling down of balanced excitation and inhibition by active behavioral states in auditory cortex. Nature Neuroscience 17, 841-850 (2014).

11. Polack, P.-O., Friedman, J. \& Golshani, P. Cellular mechanisms of brain state-dependent gain modulation in visual cortex. Nature Neuroscience 16, 1331-1339 (2013).

12. Tasic, B. et al. Shared and distinct transcriptomic cell types across neocortical areas. Nature 563, 72-78 (2018).

13. Economo, M. N. et al. Distinct descending motor cortex pathways and their roles in movement. Nature 563, 79-84 (2018).

14. Chen, J. L., Carta, S., Soldado-Magraner, J., Schneider, B. L. \& Helmchen, F. Behaviourdependent recruitment of long-range projection neurons in somatosensory cortex. Nature 499, 336-340 (2013).

15. Glickfeld, L. L., Andermann, M. L., Bonin, V. \& Reid, R. C. Cortico-cortical projections in mouse visual cortex are functionally target specific. Nat Neurosci 16, 219-226 (2013).

16. Kim, T. et al. Cortically projecting basal forebrain parvalbumin neurons regulate cortical gamma band oscillations. PNAS 112, 3535-3540 (2015).

17. Harris, K. D. \& Shepherd, G. M. G. The neocortical circuit: themes and variations. Nature Neuroscience 18, 170-181 (2015).

18. Takahashi, N. et al. Active dendritic currents gate descending cortical outputs in perception. Nature Neuroscience 1-9 (2020) doi:10.1038/s41593-020-0677-8.

19. Tang, L. \& Higley, M. J. Layer 5 Circuits in V1 Differentially Control Visuomotor Behavior. Neuron 105, 346-354.e5 (2020). 
20. Li, N., Chen, T.-W., Guo, Z. V., Gerfen, C. R. \& Svoboda, K. A motor cortex circuit for motor planning and movement. Nature 519, 51-56 (2015).

21. Chen, T.-W., Li, N., Daie, K. \& Svoboda, K. A Map of Anticipatory Activity in Mouse Motor Cortex. Neuron 94, 866-879.e4 (2017).

22. Wekselblatt, J. B., Flister, E. D., Piscopo, D. M. \& Niell, C. M. Large-scale imaging of cortical dynamics during sensory perception and behavior. Journal of Neurophysiology $\mathbf{1 1 5}$, 2852-2866 (2016).

23. Couto, J. et al. Chronic, cortex-wide imaging of specific cell populations during behavior. Nat Protoc 1-25 (2021) doi:10.1038/s41596-021-00527-z.

24. Cardin, J. A., Crair, M. C. \& Higley, M. J. Mesoscopic Imaging: Shining a Wide Light on Large-Scale Neural Dynamics. Neuron 108, 33-43 (2020).

25. Allen, W. E. et al. Global Representations of Goal-Directed Behavior in Distinct Cell Types of Mouse Neocortex. Neuron 94, 891-907.e6 (2017).

26. Matho, K. S. et al. Genetic dissection of the glutamatergic neuron system in cerebral cortex. Nature 598, 182-187 (2021).

27. Gerfen, C. R., Paletzki, R. \& Heintz, N. GENSAT BAC cre-recombinase driver lines to study the functional organization of cerebral cortical and basal ganglia circuits. Neuron $\mathbf{8 0}$, 1368-1383 (2013).

28. Harris, J. A. et al. Anatomical characterization of Cre driver mice for neural circuit mapping and manipulation. Front Neural Circuits 8, 76 (2014).

29. Daigle, T. L. et al. A Suite of Transgenic Driver and Reporter Mouse Lines with Enhanced Brain-Cell-Type Targeting and Functionality. Cell 174, 465-480.e22 (2018).

30. Wang, Q. et al. The Allen Mouse Brain Common Coordinate Framework: A 3D Reference Atlas. Cell 181, 936-953.e20 (2020).

31. Garrett, M. E., Nauhaus, I., Marshel, J. H. \& Callaway, E. M. Topography and Areal Organization of Mouse Visual Cortex. J. Neurosci. 34, 12587-12600 (2014).

32. Musall, S., Kaufman, M. T., Juavinett, A. L., Gluf, S. \& Churchland, A. K. Single-trial neural dynamics are dominated by richly varied movements. Nat Neurosci 22, 1677-1686 (2019).

33. Saxena, S. et al. Localized semi-nonnegative matrix factorization (LocaNMF) of widefield calcium imaging data. PLOS Computational Biology 16, e1007791 (2020).

34. McInnes, L., Healy, J. \& Melville, J. UMAP: Uniform Manifold Approximation and Projection for Dimension Reduction. arXiv:1802.03426 [cs, stat] (2018).

35. Brunton, B. W., Botvinick, M. M. \& Brody, C. D. Rats and Humans Can Optimally Accumulate Evidence for Decision-Making. Science 340, 95-98 (2013).

36. Salkoff, D. B., Zagha, E., McCarthy, E. \& McCormick, D. A. Movement and Performance Explain Widespread Cortical Activity in a Visual Detection Task. Cereb Cortex 30, 421-437 (2020).

37. Orsolic, I., Rio, M., Mrsic-Flogel, T. D. \& Znamenskiy, P. Mesoscale cortical dynamics reflect the interaction of sensory evidence and temporal expectation during perceptual decision-making. Neuron 109, 1861-1875.e10 (2021).

38. Steinmetz, N. A., Zatka-Haas, P., Carandini, M. \& Harris, K. D. Distributed coding of choice, action and engagement across the mouse brain. Nature 576, 266-273 (2019). 
39. Esmaeili, V. et al. Rapid suppression and sustained activation of distinct cortical regions for a delayed sensory-triggered motor response. Neuron 109, 2183-2201.e9 (2021).

40. Hanks, T. D. et al. Distinct relationships of parietal and prefrontal cortices to evidence accumulation. Nature 520, 220-223 (2015).

41. Goard, M. J., Pho, G. N., Woodson, J. \& Sur, M. Distinct roles of visual, parietal, and frontal motor cortices in memory-guided sensorimotor decisions. eLife 5, e13764 (2016).

42. Peters, A. J., Fabre, J. M. J., Steinmetz, N. A., Harris, K. D. \& Carandini, M. Striatal activity topographically reflects cortical activity. Nature 1-6 (2021) doi:10.1038/s41586-020-031668.

43. Oh, S. W. et al. A mesoscale connectome of the mouse brain. Nature 508, 207-214 (2014).

44. Mahn, M. et al. High-efficiency optogenetic silencing with soma-targeted anion-conducting channelrhodopsins. Nature Communications 9, 4125 (2018).

45. Guo, Z. V. et al. Flow of Cortical Activity Underlying a Tactile Decision in Mice. Neuron 81, 179-194 (2014).

46. MacDowell, C. J. \& Buschman, T. J. Low-Dimensional Spatiotemporal Dynamics Underlie Cortex-wide Neural Activity. Current Biology 30, 2665-2680.e8 (2020).

47. Stringer, C. et al. Spontaneous behaviors drive multidimensional, brainwide activity. Science 364, eaav7893 (2019).

48. Logothetis, N. K. et al. Hippocampal-cortical interaction during periods of subcortical silence. Nature 491, 547-553 (2012).

49. Rogers, B. P., Morgan, V. L., Newton, A. T. \& Gore, J. C. Assessing Functional Connectivity in the Human Brain by FMRI. Magn Reson Imaging 25, 1347-1357 (2007).

50. Vanni, M. P. \& Murphy, T. H. Mesoscale Transcranial Spontaneous Activity Mapping in GCaMP3 Transgenic Mice Reveals Extensive Reciprocal Connections between Areas of Somatomotor Cortex. J. Neurosci. 34, 15931-15946 (2014).

51. Vanni, M. P., Chan, A. W., Balbi, M., Silasi, G. \& Murphy, T. H. Mesoscale Mapping of Mouse Cortex Reveals Frequency-Dependent Cycling between Distinct Macroscale Functional Modules. J. Neurosci. 37, 7513-7533 (2017).

52. Macé, É. et al. Whole-Brain Functional Ultrasound Imaging Reveals Brain Modules for Visuomotor Integration. Neuron 100, 1241-1251.e7 (2018).

53. Matsui, T., Murakami, T. \& Ohki, K. Transient neuronal coactivations embedded in globally propagating waves underlie resting-state functional connectivity. PNAS 113, 6556-6561 (2016).

54. Huang, L. et al. BRICseq Bridges Brain-wide Interregional Connectivity to Neural Activity and Gene Expression in Single Animals. Cell 182, 177-188.e27 (2020).

55. Mohajerani, M. H. et al. Spontaneous cortical activity alternates between motifs defined by regional axonal projections. Nat Neurosci 16, 1426-1435 (2013).

56. Lohani, S. et al. Dual color mesoscopic imaging reveals spatiotemporally heterogeneous coordination of cholinergic and neocortical activity. 2020.12.09.418632 https://www.biorxiv.org/content/10.1101/2020.12.09.418632v1 (2020) doi:10.1101/2020.12.09.418632.

57. Gilad, A. \& Helmchen, F. Spatiotemporal refinement of signal flow through association cortex during learning. Nature Communications 11, 1-14 (2020). 
58. Gallero-Salas, Y. et al. Sensory and Behavioral Components of Neocortical Signal Flow in Discrimination Tasks with Short-Term Memory. Neuron 109, 135-148.e6 (2021).

59. Park, J. M. et al. Deep and superficial layers of the primary somatosensory cortex are critical for whisker-based texture discrimination in mice. bioRxiv 2020.08.12.245381 (2020) doi:10.1101/2020.08.12.245381.

60. Gilad, A., Gallero-Salas, Y., Groos, D. \& Helmchen, F. Behavioral Strategy Determines Frontal or Posterior Location of Short-Term Memory in Neocortex. Neuron 99, 814-828.e7 (2018).

61. Jun, E. J. et al. Causal role for the primate superior colliculus in the computation of evidence for perceptual decisions. Nat Neurosci (2021) doi:10.1038/s41593-021-00878-6.

62. Felsen, G. \& Mainen, Z. F. Neural substrates of sensory-guided locomotor decisions in the rat superior colliculus. Neuron 60, 137-148 (2008).

63. Wang, L., McAlonan, K., Goldstein, S., Gerfen, C. R. \& Krauzlis, R. J. A Causal Role for Mouse Superior Colliculus in Visual Perceptual Decision-Making. J. Neurosci. 40, 37683782 (2020).

64. Duan, C. A. et al. A cortico-collicular pathway for motor planning in a memory-dependent perceptual decision task. Nature Communications 12, 2727 (2021).

65. Hwang, E. J. et al. Corticostriatal Flow of Action Selection Bias. Neuron (2019) doi:10.1016/j.neuron.2019.09.028.

66. Erlich, J. C., Bialek, M. \& Brody, C. D. A Cortical Substrate for Memory-Guided Orienting in the Rat. Neuron 72, 330-343 (2011).

67. Zatka-Haas, P., Steinmetz, N. A., Carandini, M. \& Harris, K. D. Sensory coding and the causal impact of mouse cortex in a visual decision. eLife 10, e63163 (2021).

68. Pinto, L. et al. Task-Dependent Changes in the Large-Scale Dynamics and Necessity of Cortical Regions. Neuron 104, 810-824.e9 (2019).

69. Kim, J.-H., Ma, D.-H., Jung, E., Choi, I. \& Lee, S.-H. Gated feedforward inhibition in the frontal cortex releases goal-directed action. Nat Neurosci 24, 1452-1464 (2021).

70. Wu, Z. et al. Context-Dependent Decision Making in a Premotor Circuit. Neuron 106, 316328.e6 (2020).

71. Coen, P., Sit, T. P. H., Wells, M. J., Carandini, M. \& Harris, K. D. Mouse frontal cortex mediates additive multisensory decisions. 2021.04.26.441250

https://www.biorxiv.org/content/10.1101/2021.04.26.441250v2 (2021)

doi:10.1101/2021.04.26.441250.

72. Park, J., Phillips, J. W., Martin, K. A., Hantman, A. W. \& Dudman, J. T. Descending neocortical output critical for skilled forelimb movements is distributed across projection cell classes. $772517 \mathrm{https}$ //www.biorxiv.org/content/10.1101/772517v3 (2021) doi:10.1101/772517.

73. Znamenskiy, P. \& Zador, A. M. Corticostriatal neurones in auditory cortex drive decisions during auditory discrimination. Nature 497, 482-485 (2013).

74. Laboulaye, M. A., Duan, X., Qiao, M., Whitney, I. E. \& Sanes, J. R. Mapping Transgene Insertion Sites Reveals Complex Interactions Between Mouse Transgenes and Neighboring Endogenous Genes. Frontiers in Molecular Neuroscience 11, 385 (2018).

75. Callaway, E. M. et al. A multimodal cell census and atlas of the mammalian primary motor cortex. Nature 598, 86-102 (2021). 
76. Ratzlaff, E. H. \& Grinvald, A. A tandem-lens epifluorescence macroscope: hundred-fold brightness advantage for wide-field imaging. J. Neurosci. Methods 36, 127-137 (1991).

77. Lerner, T. N. et al. Intact-Brain Analyses Reveal Distinct Information Carried by SNc Dopamine Subcircuits. Cell 162, 635-647 (2015).

78. Marshel, J. H., Garrett, M. E., Nauhaus, I. \& Callaway, E. M. Functional Specialization of Seven Mouse Visual Cortical Areas. Neuron 72, 1040-1054 (2011).

79. Chuong, A. S. et al. Noninvasive optical inhibition with a red-shifted microbial rhodopsin. Nat Neurosci 17, 1123-1129 (2014).

80. Reddy, B. S. \& Chatterji, B. N. An FFT-based technique for translation, rotation, and scaleinvariant image registration. IEEE Trans Image Process 5, 1266-1271 (1996).

81. Karabatsos, G. Marginal maximum likelihood estimation methods for the tuning parameters of ridge, power ridge, and generalized ridge regression. Communications in Statistics Simulation and Computation (2017). 